\title{
Multiresolution Analysis Adapted to Irregularly Spaced Data
}

\author{
Anissa Mokraoui ${ }^{1}$ and Pierre Duhamel (EURASIP Member) ${ }^{2}$ \\ ${ }^{1}$ L2TI, Institut Galilée, Université Paris 13, 99, Avenue Jean Baptiste Clément, 93430 Villetaneuse, France \\ ${ }^{2}$ LSS/CNRS, SUPELEC, Plateau de Moulon, 91192 Gif sur Yvette, France
}

Correspondence should be addressed to Anissa Mokraoui, anissa.mokraoui@univ-paris13.fr

Received 26 February 2009; Revised 18 July 2009; Accepted 18 September 2009

Recommended by Enrico Capobianco

This paper investigates the mathematical background of multiresolution analysis in the specific context where the signal is represented by irregularly sampled data at known locations. The study is related to the construction of nested piecewise polynomial multiresolution spaces represented by their corresponding orthonormal bases. Using simple spline basis orthonormalization procedures involves the construction of a large family of orthonormal spline scaling bases defined on consecutive bounded intervals. However, if no more additional conditions than those coming from multiresolution are imposed on each bounded interval, the orthonormal basis is represented by a set of discontinuous scaling functions. The spline wavelet basis also has the same problem. Moreover, the dimension of the corresponding wavelet basis increases with the spline degree. An appropriate orthonormalization procedure of the basic spline space basis, whatever the degree of the spline, allows us to (i) provide continuous scaling and wavelet functions, (ii) reduce the number of wavelets to only one, and (iii) reduce the complexity of the filter bank. Examples of the multiresolution implementations illustrate that the main important features of the traditional multiresolution are also satisfied.

Copyright ( 92009 A. Mokraoui and P. Duhamel. This is an open access article distributed under the Creative Commons Attribution License, which permits unrestricted use, distribution, and reproduction in any medium, provided the original work is properly cited.

\section{Introduction}

Multiresolution theory has been extensively studied for more than a decade (see, e.g., [1-4]). Initially, the multiresolution theory has been mainly developed within the framework of samples taken at regular sampling instants. Therefore the scaling and wavelet bases are built under the assumptions that the knots associated to the discrete signal are regularly spaced. The scaling or wavelet basis is defined as a set of translations and dilations of a single prototype function. Thus, the obtained functions are similar to each other at different scales.

However, the nonuniform sampling situation arises naturally in many scientific fields such as geophysics, astronomy, meteorology, medical imaging and computer vision where data are often generated or measured at sparse and irregular known positions. In the literature few works are available in this context, compared to those developed in the case of regularly spaced data. It is within this framework that we have concentrated our study. The proposed approach can be used to interpolate irregularly sampled signals in an efficient way, by keeping the multiresolution strategy.
The nonequally spaced data assumption results in a more general definition of the scaling and wavelet functions. The development of the scaling and wavelet bases, provided in this paper, focuses on piecewise polynomials, named nonuniform B-spline functions. These functions are widely used to model curves and surfaces in computer graphics [5]. The spline functions were already successfully used in the uniform spacing knot case leading to the construction of spline scaling and wavelet functions. An extensive bibliography is available (see, e.g., [6-10]), while relatively little works have been published about the construction of these functions on arbitrary nonuniformly spaced knots.

The construction of the scaling and wavelet bases on irregular spacing knots is more complicated than the traditional case (equally spaced knots). On a nonequally spaced knots sequence, previous works (see, e.g., [11-18]) show that the underlying concept of dilating and translating one unique prototype function which allows the construction of the scaling and wavelet bases is not valid any more. Some important previous works, in relation to the subject of our investigation are briefly summarized in the following pages. 
The authors of [11], review and discuss some techniques and tools for constructing wavelets on an irregular set of points by means of generalized subdivision schemes and commutation rules. They have adapted Lemariés commutation formula to an irregular setting [12]. Starting from a coarse and irregular set of points, the subdivision technique consists in iterating the upsampling process using a local averaging function to refine and control the shape of a curve. In this case, the wavelet and scaling functions from the coarsest level are generated with a subdivision scheme using new points provided by the finest level grid on which the data was initially sampled. However, smoothness results in these settings become much harder to obtain.

This strategy has been widely used in the two dimensional case based on the triangulation plane of a function [19] which is also based on the formalism of a commutation approach for an irregular subdivision scheme. The authors of reference [13] proposed as a sequel of paper [11] the construction of an entire family of biorthogonal compactly supported irregular knot B-spline wavelets.

In reference [15], Buhmann and Micchelli originally presented a theoretical study to perform a multiresolution analysis using spline spaces of arbitrary degree adapted to nonuniform knot sequences. They proposed a generalization of the cardinal spline approach to the wavelets which have been extensively studied in the uniform spaced data case [3]. The proposed wavelet (named prewavelet in their paper) is given as the $(n+1)$ th order derivative of the spline function of degree $2 n+1$. The existence of compactly supported wavelets which decay exponentially has been shown. The support of the wavelet depends on the degree $n$ of the spline function and is given by the interval $\left[x_{i}, x_{i+2 n+1}\right]$ (where $x_{k}$ specifies the data position). Moreover, Buhmann and Micchelli proved that the insertion of $L$ multiple knots between two consecutive knots belonging to the previous coarse scale involves $2 n+L$ wavelets.

On another hand, the authors of paper [14] investigated the construction of a semiorthogonal spline wavelet basis for nonuniform partitions on a bounded interval. They proposed the construction of nonuniform B-spline functions with multiple knots at each end point of the interval as special boundary functions. They provided explicit expressions of the nonuniform B-spline wavelets. Decomposition and reconstruction algorithms using filter banks are also proposed. However the dimension of the spline wavelet space increases with the insertion of knots from one resolution level to another. This situation increases the computational complexity of the filter bank and moreover its implementation.

In [17], Wang focused on the construction of compactly supported wavelets on arbitrary partitions where additional conditions were imposed on the end-points of the cubic spline thereby obtaining a unique interpolating cubic spline.

In [20], the spline basis orthonormalization process used for constructing the orthonormal scaling and wavelet bases, is defined separately on each bounded interval of the knots sequence. This process introduces discontinuities of the spline scaling functions at each end-point of the intervals.
The resulting spline wavelet basis functions are also affected. The wavelet functions are not only discontinuous at each end-point of the nonuniform B-spline intervals but are also inside each interval. Moreover, the dimension of the spline wavelet space is proportional to the degree of the spline function.

In the framework of this paper, we have chosen to work separately on each bounded interval of the real line and to impose multiplicities of maximal order at end-points of the B-spline function definition domain [21]. The generated piecewise polynomial spaces allow an obvious scaling of the spaces as required for a multiresolution construction. Indeed, a piecewise polynomial of a given degree over a bounded interval at any resolution level is also a piecewise polynomial at another resolution level. Moreover, many simple bases can be built for such piecewise polynomial spaces.

The main objective of this paper is to propose a basis orthonormalization procedure which allows for the construction of suitable bases of the spline scaling and wavelet spaces ensuring the continuities of all functions whatever the degree of the spline function. By doing so, some drawbacks inherent to the developed methods briefly described above are improved. Whatever the partition of the knots, we provide a generalization of the multiresolution approach compared to the works provided in $[11,19]$.

In these references the process begins with a coarse irregular subdivision where the refinement step is semiregular (i.e., the point is inserted in the middle of two consecutive knots). In the present paper the support of the wavelet function at each resolution level is fixed by the initial partition of the knots, while in [15] it depends on the spline function degree. Moreover, we show in the next sections that the computational complexity is significantly reduced because the number of spline wavelets is reduced to only one, whatever the spline degree, while in [15] the number of the wavelets depends on the inserted knots and the fixed derivative order of the cardinal spline function. In paper [14], the number of semi-orthogonal spline wavelet functions increases with the refinement steps.

This paper is organized as follows. Section 2 focuses on the multiresolution analysis concepts yielding to the construction of the spline scaling and wavelet subspaces. Section 3 describes the new spline scaling basis orthonormalization procedure, which can be applied whatever the degree of the spline function. Finally, Section 4 is concerned with the construction of the orthonormal spline wavelet basis, while the corresponding orthogonal decomposition scheme is developed in Section 5. Multiresolution implementation results are then discussed in Section 6 followed by Section 7 which concludes the work.

\section{Concepts for the Multiresolution Analysis Adapted to Irregularly Sampled Data}

This section focuses on the multiresolution analysis concepts according to the nonequally sampled discrete signal. Before we are going to introduce the basic definitions and properties 
of the nonuniform B-spline function necessary for our later developments.

2.1. Basic Nonuniform Spline Space. Among the large family of polynomials, the nonuniform B-spline functions have been selected in this paper since they provide many interesting properties which will be used later for the multiresolution approach [21].

Let start with some notations. The knots sequence is composed of known knots corresponding to the locations of the available data representing the discrete signal. This knots sequence is organized according to an increasing order:

$$
t_{0}<t_{1}<\cdots<t_{i}<t_{i+1}<\cdots \quad \forall i \in \mathbb{N} .
$$

The nonuniform B-spline function definition has been initially proposed by Curry and Schoenberg [22]. Given a set of $d+2$ samples located at arbitrary known knots. The $i$ th nonuniform B-spline function of degree $d$, denoted by $B_{i,\left[t_{i}, t_{i+d+1}\right]}^{d}(t)$, is considered as a piecewise polynomial of degree $d$ defined on a compact support $\left[t_{i}, t_{i+d+1}\right]$. As originally proposed in [21], it is given by the following formula:

$$
B_{i,\left[t_{i}, t_{i+d+1}\right]}^{d}(t)=\left(t_{i+d+1}-t_{i}\right)\left[t_{i}, \ldots, t_{i+d+1}\right](\cdot-t)_{+}^{d},
$$

where $(x-t)_{+}=\max (x-t, 0)$ represents the truncated power function and $\left[t_{i}, t_{i+d+1}\right]$ is the $(d+1)$ th divided difference operator applied to the function $(\cdot-t)_{+}^{d}$. Remember that the divided difference operator is defined as follows:

$$
\begin{aligned}
& {\left[t_{i}, \ldots, t_{i+d+1}\right](\cdot-t)_{+}^{d} } \\
&=\left(t_{i+d+1}-t_{i}\right)^{-1}\left(\left[t_{i+1}, \ldots, t_{i+d+1}\right](\cdot-t)_{+}^{d}\right. \\
&\left.\quad-\left[t_{i}, \ldots, t_{i+d}\right](\cdot-t)_{+}^{d}\right) .
\end{aligned}
$$

If any arbitrary knot $t_{k}$, belonging to the sequence $t_{i}<\cdots<$ $t_{i+d+1}$, has a multiplicity of order $\mu_{k}+1$ (i.e., the knot occurs $\mu_{k}+1$ times) then the divided difference definition (see (3)) applied to the function $g=(\cdot-t)_{+}^{d}$ becomes

$$
\left[t_{0}, \ldots, t_{\mu_{k}}\right] g=\frac{g^{\mu_{k}}\left(t_{0}\right)}{\mu_{k} !} \quad \text { if } t_{0}=\cdots=t_{\mu_{k}} .
$$

The number of times $\left(r_{k}-1\right)$ that the B-spline function is continuously differentiable at the knot $t_{k}$ is directly related to the multiplicity $\left(\mu_{k}+1\right)$ imposed on the knot $t_{k}$ :

$$
r_{k}+\mu_{k}=d+1
$$

Thus, the B-spline function regularity is $C^{d-\mu_{k}}$. It was proven in reference [21] that the $n$ nonuniform B-spline functions set $\left\{B_{i,\left[t_{i}, t_{i+d+1}\right]}^{d}, \ldots, B_{i+n-1,\left[t_{i+n-1}, t_{i+n+d}\right]}^{d}\right\}$ defined on the knots sequence $a=t_{i}<t_{i+1}<\cdots<t_{i+d+n}=b$, generates a basis for the piecewise polynomials space of degree $d$. The spline space restricted to the interval $[a, b]$ is a closed subspace of $L_{2}(\mathbb{R})$ and is denoted $V_{0}^{d}[a, b]$. The dimension $n$ of the spline basis depends on the multiplicities imposed on each knot of the sequence [21]. Hence, for a fixed degree of the spline function, several bases of various dimensions can be constructed for the corresponding piecewise polynomial space.

In previous work we studied the influence of the dimension of the spline basis on the interpolation error. The comparison of the upper bounds of the interpolation error shows that the smallest one is given for the smallest dimension, that is, $d+1$ [18]. This imposes a multiplicity of order $d+1$ on each knot of the sequence. Thus, the B-spline functions are defined between two consecutive knots of the considered sequence. This strategy is generally used to construct special boundary functions in an interval. These particular B-splines are also known in the literature as Bernstein polynomials when the bounded interval is restricted to $[\ldots, 0,0,1,1, \ldots]$. The corresponding basis is well know in CAGD as the Bernstein-Bézier Form (BBF). Our study is based on this configuration of knots.

In the following sections, any knot of multiplicity order $d+1$ is denoted indifferently $t_{i}$ or $\tau_{i}^{d+1}$. Whatever the spline degree, the generalized expression of the nonuniform Bspline function is given by $[5,21]$

$$
\begin{array}{r}
B_{k,\left[t_{i}, t_{i+d+1}\right]}^{d}(t)=C_{d}^{k}\left(\frac{t_{i+1}-t}{t_{i+1}-t_{i}}\right)^{d-k}\left(\frac{t-t_{i}}{t_{i+1}-t_{i}}\right)^{k} \\
\quad \text { for } t_{i} \leq t \leq t_{i+1}, \quad 0 \leq k \leq d \quad \forall i \in \mathbb{N},
\end{array}
$$

where $C_{d}^{k}$ is the binomial coefficient. The basic spline subspace of dimension $d+1$, denoted $\mathbb{S}_{0}^{d}\left[t_{i}, t_{i+1}\right]$, is defined as follows:

$$
\begin{aligned}
\mathbb{S}_{0}^{d}\left[t_{i}, t_{i+1}\right]= & \left\{f: f(t)=\sum_{k=0}^{d} a_{k,\left[t_{i}, t_{i+1}\right]} B_{k,\left[t_{i}, t_{i+1}\right]}^{d}(t)\right. \\
& \text { for } \left.t_{i} \leq t \leq t_{i+1}, a_{k,\left[t_{i}, t_{i+1}\right]} \in l_{2}\right\} \quad \forall i \in \mathbb{N},
\end{aligned}
$$

where $f(t)$ is the spline function of degree $d$ and the set $\left\{a_{k,\left[t_{i}, t_{i+1}\right]}\right\}$ represents the B-spline coefficients of the spline function. The basic spline space of the global sequence, denoted $\mathbb{S}_{0}^{d}$, is given as the union of the closed subspaces as follows:

$$
\mathbb{S}_{0}^{d}=\bigcup_{i=0}^{\infty} \mathbb{S}_{0}^{d}\left[t_{i}, t_{i+1}\right] .
$$

Figure 1 presents the spline basis elements of the basic space $\mathbb{S}_{0}^{d}[0,2]$ for four spline degrees $d=1, d=2, d=3$ and $d=4$ defined on the bounded interval $\left[\tau_{0}^{d+1}=0, \tau_{1}^{d+1}=2\right]$. Let us point out that the spline basis functions are naturally symmetrical by pair, in reference to the coordinate of the mid point $t_{s_{i}}=\left(t_{i}+t_{i+1}\right) / 2$, except for this single function $B_{d,\left[t_{i}, t_{i+1}\right]}^{d}(t)$ when the spline degree is even. This last function is itself symmetric with respect to $t_{s_{i}}$. Therefore the set of $\lfloor(d-1) / 2\rfloor+1$ B-spline functions are easily deduced as follows:

$$
\begin{array}{r}
B_{d-k,\left[t_{i}, t_{i+1}\right]}^{d}(t)=B_{k,\left[t_{i}, t_{i+1}\right]}^{d}\left(t_{i}+t_{i+1}-t\right) \\
\text { for } k=0, \ldots,\left\lfloor\frac{(d-1)}{2}\right\rfloor,
\end{array}
$$




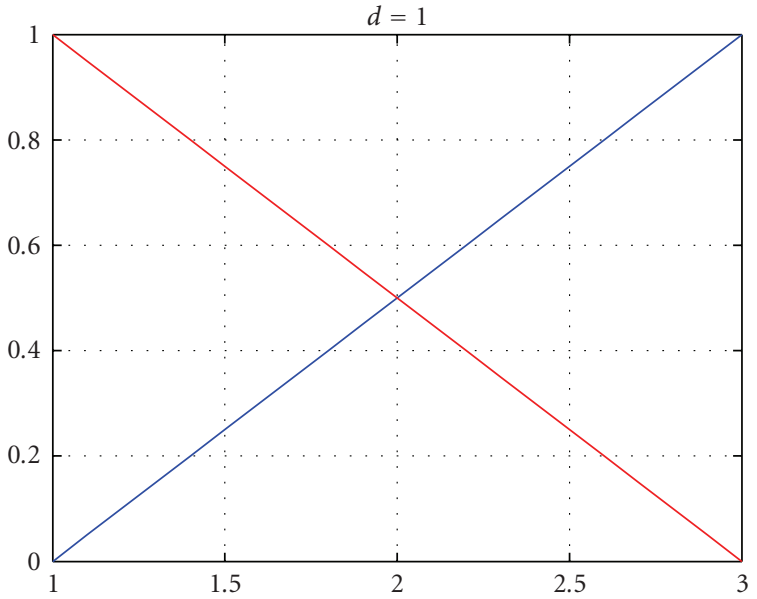

(a)

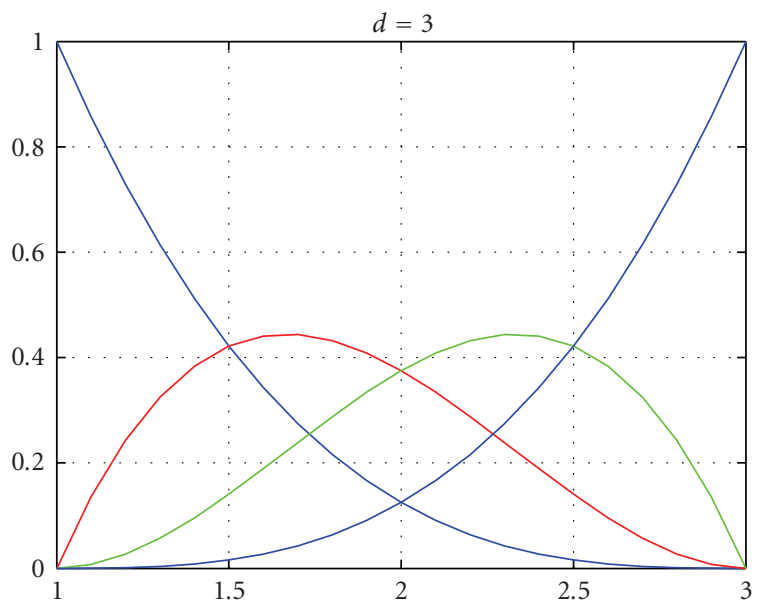

(c)

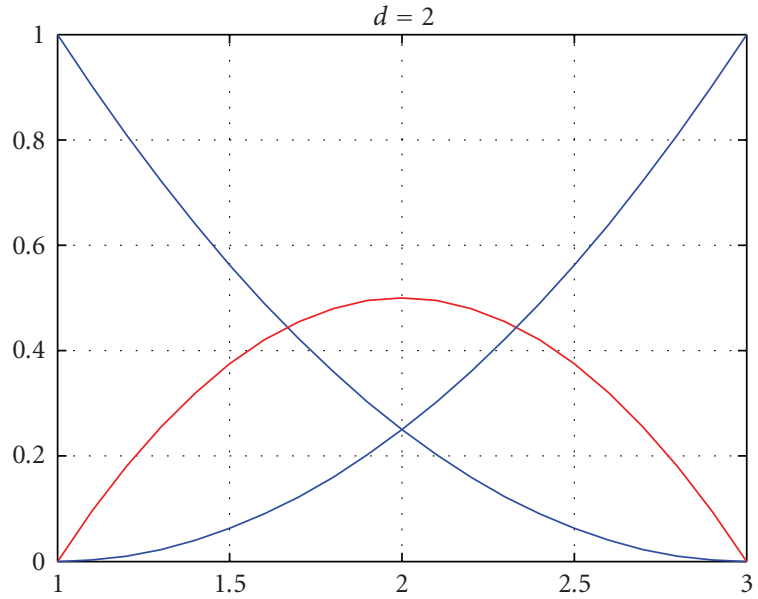

(b)

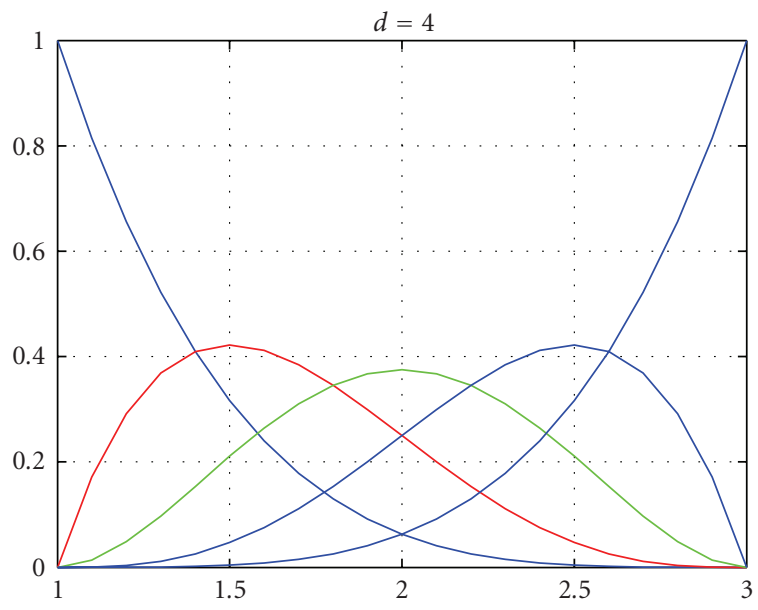

(d)

FIGURE 1: Nonuniform spline bases for $d=1, d=2, d=3$ and $d=4$.

where $L \cdot\rfloor$ is the floor function. These suitable properties are emphasized since they will be used in what follows.

2.2. Multiresolution Analysis Concepts. Classically, a multiresolution analysis consists of approximating a given signal at different resolution levels, and of providing recurrence relations which allows to go from one resolution to the next (see, e.g., [1-4]).

Let us assume that the nonequally spaced knots partition, given by (1), is a finite sequence $t_{0}<t_{1}<\cdots<t_{i}<$ $t_{i+1}<\cdots<t_{N}$, where $N$ represents an integer multiple of $2^{J}$ with $J$ corresponding to a fixed lower resolution level. This sequence, denoted $S_{0}$, is considered as the finest sequence where a multiplicity of order $\mu=d+1$ is imposed at each knot of the sequence. We should first introduce the bounded interval, denoted $I_{j, i}$ at any given resolution level $j$, as follows:

$$
I_{j, i}=\left[t_{2^{j} i}, t_{2^{j}(i+1)}\right]=\left[\tau_{2^{j} i}^{d+1}, \tau_{2^{j}(i+1)}^{d+1}\right] \quad \forall i, j \in \mathbb{N} .
$$

At any resolution level $j$, the corresponding sequence $S_{j}$ is thus built from the union of bounded intervals $I_{j, i}$ as defined below:

$$
S_{j}=\bigcup_{i=0}^{N-1} I_{j, i} \quad \forall i, j \in \mathbb{N} .
$$

Going from the resolution level $j-1$ (fine resolution) to the resolution level $j$ (coarse resolution of scale) consists of removing one knot out of two in the sequence $S_{j-1}$. This is obtained by means of several intermediate steps in removing $d+1$ multiplicities on these knots. This results in a set of embedded sub-sequences as follows:

$$
S_{0} \supset S_{1} \cdots \supset S_{j-1} \supset S_{j} \supset \cdots \supset S_{J} .
$$

The approximation of the signal $y(t)$ at resolution level $j$, on each bounded interval $I_{j, i}$, is denoted $y_{I_{j, i}}(t)$. In order to minimize the approximation error $\left(\left\|y(t)-y_{I_{j, i}}(t)\right\|\right)$, the approximation of the signal $y(t)$ at resolution level $j$ is traditionally defined as its orthogonal projection on 


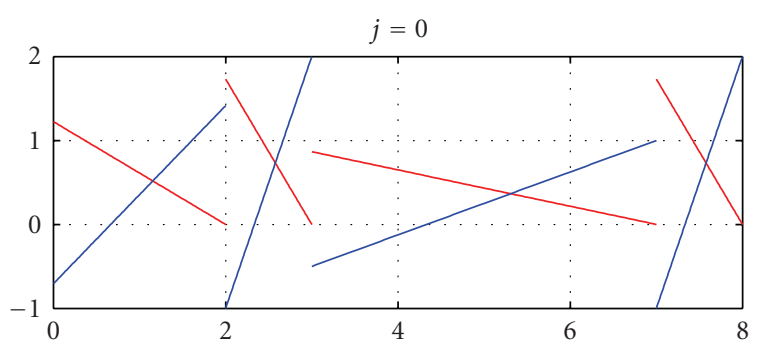

(a)

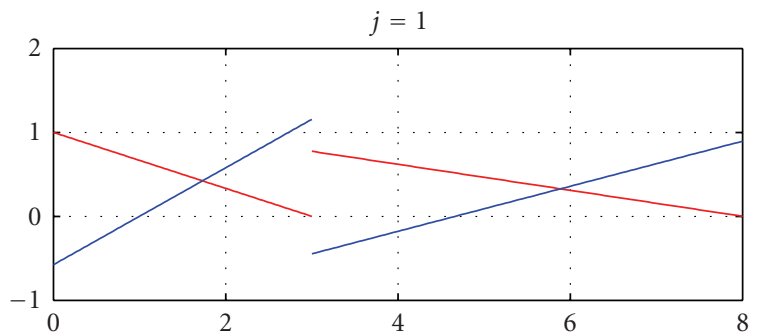

(b)

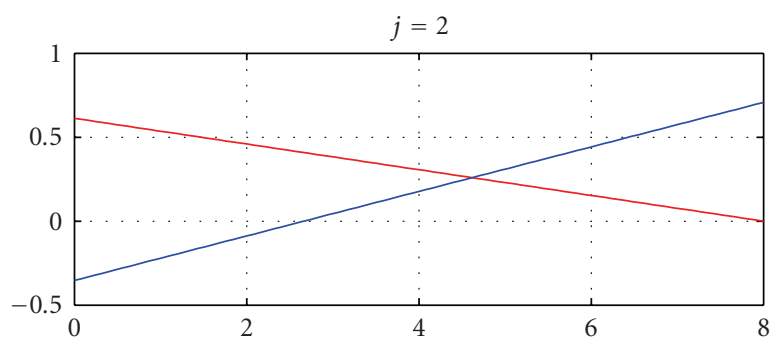

(c)

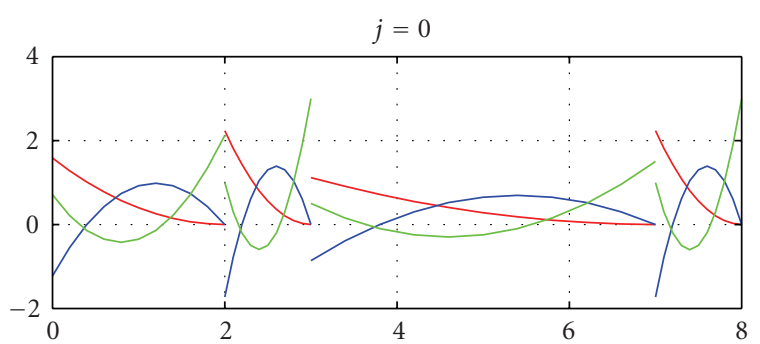

(a)

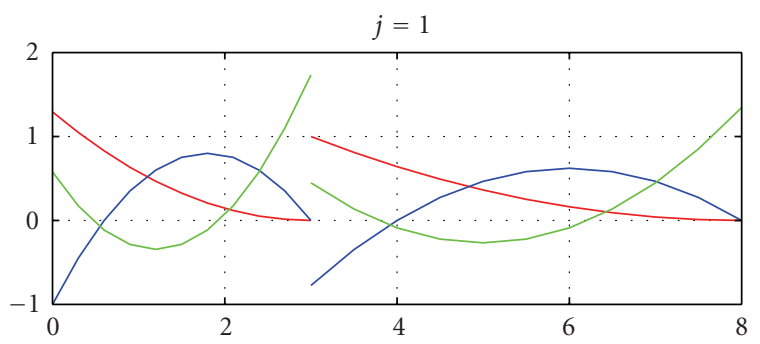

(b)

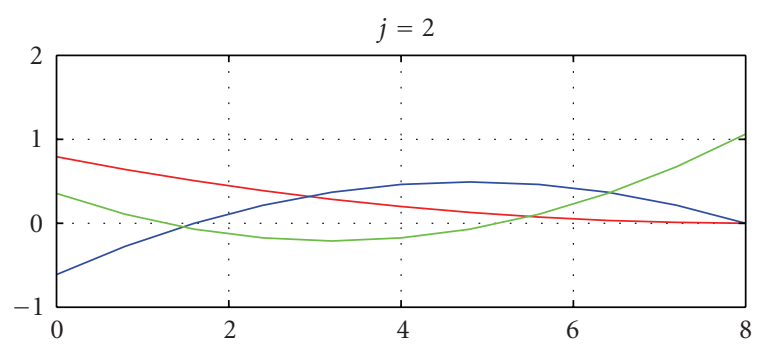

(c)

FIGURE 2: Orthonormal linear (left graphs) and quadratic (right graphs) piecewise polynomial scaling functions at $j=0,1,2$.

the subspace on which it belongs. This subspace, which will be defined later, is known as its approximation or scaling subspace.

In this paper, the functions belonging to the new basic space, denoted $\mathbb{V}_{0}^{d}$, is nothing other than a subspace of piecewise polynomials of degree $d$ over each bounded interval $I_{0, i}$. Satisfying some additional properties as it will be described in the following section. Due to the specific embedded subsequence structure (12), the functions defined on any interval $I_{j, i}$ are therefore obviously piecewise polynomials of degree $d$. Consequently, the new scaling subspaces are also nested as follows:

$$
\mathbb{V}_{0}^{d} \supset \mathbb{V}_{1}^{d} \cdots \supset \mathbb{V}_{j-1}^{d} \supset \mathbb{V}_{j}^{d} \supset \cdots \mathbb{V}_{J}^{d}
$$

where $\mathbb{V}_{j}^{d}=\bigcup_{i=0}^{N-1} \mathbb{V}_{j}^{d}\left[I_{j, i}\right]$ for all $j \in \mathbb{N}$.

The successive approximations, on each bounded interval, of any signal at two successive resolutions $j-1$ and $j$ are obtained from the orthogonal projections on the respective approximation subspaces $\mathbb{V}_{j-1}^{d}\left[I_{j-1, i}\right] \cup \mathbb{V}_{j-1}^{d}\left[I_{j-1, i+1}\right]$ and $\mathbb{V}_{j}^{d}\left[I_{j, i}\right]$. In order to represent the necessary "details" which allow us to improve the signal approximation from subspace $\mathbb{V}_{j}^{d}\left[I_{j, i}\right]$ to subspace $\mathbb{V}_{j-1}^{d}\left[I_{j-1, i}\right] \cup \mathbb{V}_{j-1}^{d}\left[I_{j-1, i+1}\right]$, one introduces the orthogonal complement of subspace $\mathbb{V}_{j}^{d}\left[I_{j, i}\right]$ in subspace $\mathbb{V}_{j-1}^{d}\left[I_{j-1, i}\right] \cup \mathbb{V}_{j-1}^{d}\left[I_{j-1, i+1}\right]$. The orthogonal subspace, known as detail or wavelet subspace is denoted by $\mathbb{W}_{j}^{d}\left[I_{j, i}\right]$. Hence, we have

$$
\begin{array}{r}
\mathbb{V}_{j-1}^{d}\left[I_{j-1, i}\right] \bigcup \mathbb{V}_{j-1}^{d}\left[I_{j-1, i+1}\right]=\mathbb{V}_{j}^{d}\left[I_{j, i}\right] \bigoplus \mathbb{W}_{j}^{d}\left[I_{j, i}\right] \\
\forall j \geq 1 .
\end{array}
$$

Since the approximation subspaces $\mathbb{V}_{j}^{d}\left[I_{j, i}\right]$ are spanned by specific piecewise polynomials, the detail subspace $\mathbb{W}_{j}^{d}\left[I_{j, i}\right]$ is also a piecewise polynomial wavelet subspace at any resolution level $j$.

In order to meet the classical conditions used in a multiresolution approach, this paper concentrates on the orthogonalization of the nonuniform spline basis of the basic spline space $\mathbb{S}_{0}^{d}$. The normalization of the orthogonal basis is also an important step since the normalization factor maintains the same signal energy at each scale. Hence the new basic piecewise polynomial space $\mathbb{V}_{0}^{d}$ is defined on the real line $S_{0}$, as follows:

$$
\mathbb{V}_{0}^{d}=\left\{f: f(t)=\sum_{k} c_{k} \underline{\varphi}_{k}^{d}(t) \text { for } c_{k} \in l_{2}, t \in S_{0}\right\},
$$

where the set $\left\{\underline{\varphi}_{k}^{d}(t)\right\}$ represents the orthonormal scaling basis functions which will be constructed in Sections 5 and 6 . 

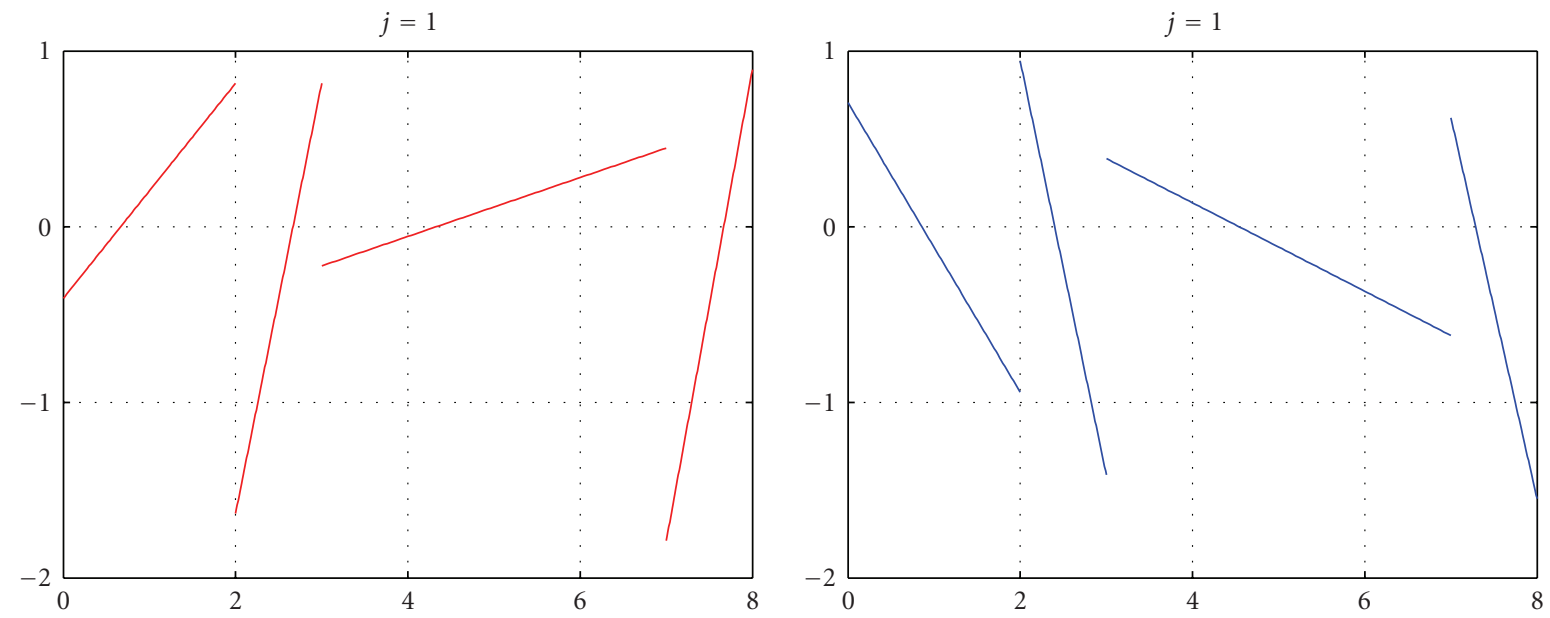

(a)
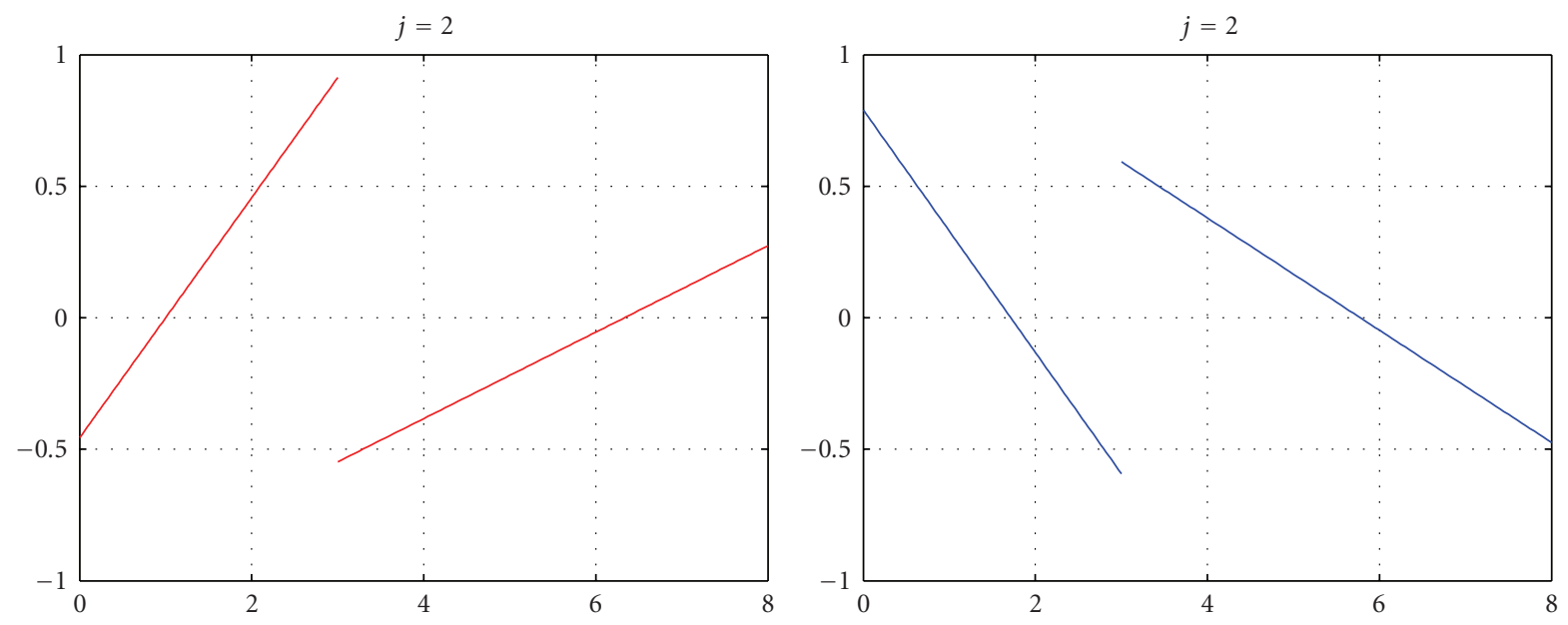

(b)

Figure 3: Orthonormal linear spline wavelet basis at resolution levels $j=1,2$.

Our study shows that there are several possible ways of orthonormalizing the nonuniform spline basis of the basic space $\mathbb{V}_{0}^{d}$. Before presenting the new basis orthonormalization procedure, Section 2.3 summarizes the previous procedure described in [20] and points out some weaknesses of the resulting scaling and wavelet functions.

\subsection{Review of the Previous Orthonormalization Procedure.} In the previous orthonormalization procedure provided in [20], the classical Gram-Schmidt method has been applied to orthonormalize the nonuniform spline basis of the basic spline space $\mathbb{S}_{0}^{d}\left[I_{0, i}\right]$, separately on each bounded interval $I_{0, i}$. A large family of orthonormal spline scaling basis can be constructed since the Gram-Schmidt method allows us to choose various functions as the reference one, thus generating several bases.

As an example of the previous orthonormalization method, Figure 2 presents the linear and quadratic orthonormal spline scaling basis functions carried out on the initial finest knot sequence $S_{0}=\left[\tau_{0}^{d+1}=0, \tau_{1}^{d+1}=2, \tau_{2}^{d+1}=\right.$ $\left.3, \tau_{3}^{d+1}=7, \tau_{4}^{d+1}=8\right]$. The left graphs correspond to the linear case while the right ones correspond to the quadratic case. Three resolution levels are plotted for $j=0$ (graphs (a)), $j=1$ (graphs (b)) and $j=2$ (graphs (c)). Figure 2 clearly shows that the constructed scaling functions, at any resolution level $j$, are not continuous at the end-points of adjacent intervals of the knots sequence $S_{j}$. However, the continuity feature for many applications is considered as an important characteristic to be satisfied.

The resulting orthonormal wavelet basis developed in [5] is constructed according to the specific traditional multiresolution conditions applied independently on each of the knot sequence's bounded intervals without imposing any additional condition. The dimension of the wavelet subspace $\left(\mathbb{W}_{j}^{d}\left[I_{j, i}\right]\right)$ on each bounded interval $I_{j, i}$ is equal to $d+1$. Figure 3 presents an example of the two linear polynomial wavelet functions at resolution levels $j=1$ (graphs (a)) and $j=2$ (graphs (b)), on the preceding finest sequence $S_{0}$. This example shows that the wavelet functions are neither continuous, that is to say, inside the bounded interval on which they belong, nor at the end-points of two adjacent intervals. 


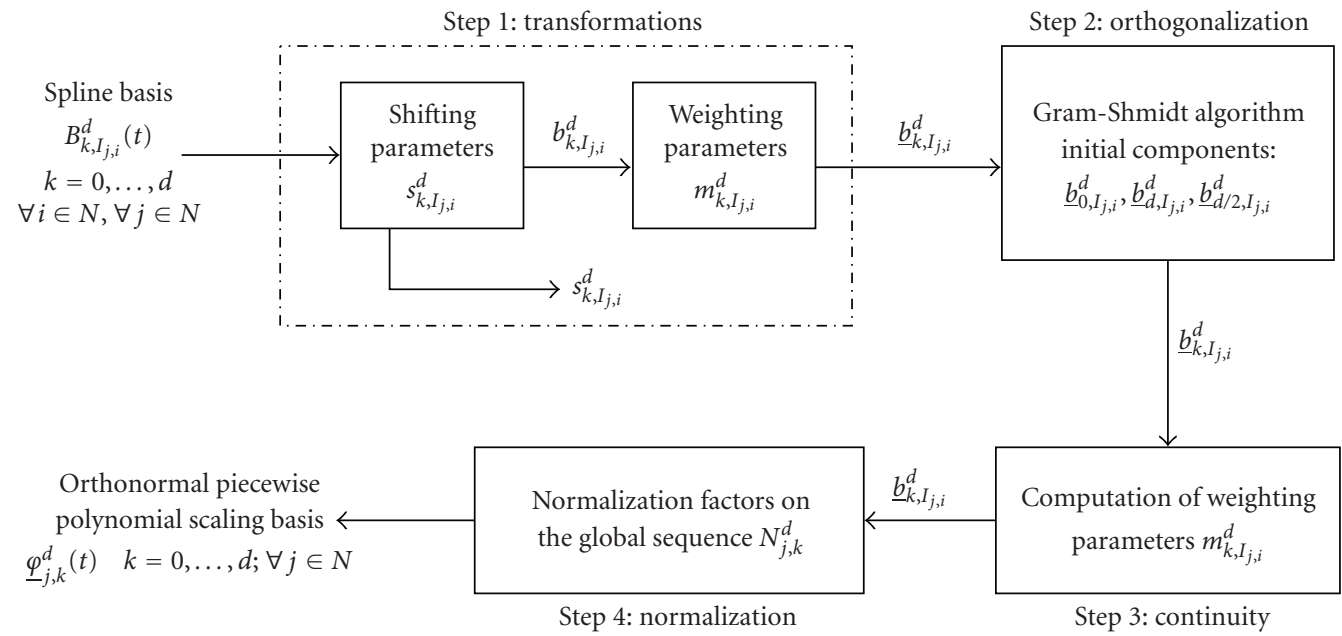

FIGURE 4: Global orthonormalization scheme.

\section{Piecewise Polynomial Scaling Basis Using New Orthonormalization Procedure}

The lack of continuity, as shown in the previous section, is clearly a drawback in many applications. Indeed the approximation error may increase particularly at the points where the fonction presents discontinuities. In some cases, this situation can influence application performances. This section proposes a unified construction of the orthonormal basis of the basic scaling space $\mathbb{V}_{0}^{d}$ in such a way that the functions of the basis are continuous at the end-points of consecutive intervals.

In this paper, the orthonormalization procedure strategy is mainly based on the introduction of additional freedom parameters (named shifting and weighting parameters) on which the procedure controls the partial orthogonality and the continuity of the scaling functions at each end-point of their definition domain. A gradual orthonormalization procedure is proposed where the main steps are summarized by the global scheme in Figure 4 .

The procedure is divided into four main parts (see Figure 4). The first one corresponds to pre-processing transformations applied to the spline elements, the second one considers the transformed spline basis orthogonalization problem, the third one deals with continuity and the last one is concerned with the normalization. In order for the presentation to be as general as possible, the basis is directly constructed for any scaling subspace $\mathbb{V}_{j}^{d}$, based on the property that the scaling subspaces are nested (see (13)).

\subsection{First Step: Transformations Using Shifting and Weighting} Parameters. Rather than working directly on the initial elements of the spline basis, as suggested in [20], we propose a pre-processing step. This step is a partial orthogonalization procedure which ensures two by two orthogonality and the continuity of the extreme and central transformed B-spline functions. This strategy is based on the remark pointed out in Section 2 that the spline basis functions are symmetrical by pair, except for one B-spline if the degree of the piecewise polynomial is even (see Figure 1 and (9)). In this context, one can imagine a large family of transformations preserving the property of a basis. However, in this paper the choice is guided by transformations which (i) maintain the initial shapes of the spline elements, and (ii) reduce the number of parameters (controlling the continuity) to be updated during the multiresolution analysis. Trying to be in accordance with points (i) and (ii), we propose the following transformations. The initial B-splines are transformed by shifting parameters $\left(\left\{s_{k, I_{j, i}}^{d}\right\}\right)$ and weighting parameters $\left(\left\{m_{k, I_{j, i}}^{d}\right\}\right)$ as described above.

3.1.1. Shifting Parameters. Each of the $d+1$ initial nonuniform B-spline functions $\left\{B_{k, I_{j, i}}^{d}(t)\right\}$ of (7) follows shift transformations provided by (16), (17) and (18):

$$
\begin{aligned}
b_{k, I_{j, i}}^{d}(t)=s_{k, I_{j, i}}^{d}+B_{k, I_{j, i}}^{d}(t) & \text { for } k=0, k=d, \\
b_{k, I_{j, i}}^{d}(t)=s_{k, I_{j, i}}^{d}+B_{k, I_{j, i}}^{d}(t), & b_{k, I_{j, i}}^{d-k}(t)=-s_{k, I_{j, i}}^{\mathrm{d}}+B_{k, I_{j, i}}^{d-k}(t) \\
\text { for } k & =1, \ldots,\left\lfloor\frac{(d-1)}{2}\right\rfloor,
\end{aligned}
$$

where $\lfloor\cdot\rfloor$ is the floor function. Moreover, when $d$ is even, one additional transformation is applied as follows:

$$
b_{k, I_{j, i}}^{d}(t)=s_{k, I_{j, i}}^{d}+B_{k, I_{j, i}}^{d}(t), \quad \text { for } k=\frac{d}{2} .
$$

In the first step, the shifting parameters are computed in order to ensure, in each bounded interval of the knot sequence $S_{j}$, the orthogonality conditions between

(i) all functions of (16) and (18):

$$
\begin{gathered}
\left\langle b_{0, I_{j, i}}^{d}(t), b_{d / 2, I_{j, i}}^{d}(t)\right\rangle=0 ; \quad\left\langle b_{0, I_{j, i}}^{d}(t), b_{d, I_{j, i}}^{d}(t)\right\rangle=0, \\
\left\langle b_{d, I_{j, i}}^{d}(t), b_{d / 2, I_{j, i}}^{d}(t)\right\rangle=0 \quad \forall i, j \in \mathbb{N}
\end{gathered}
$$




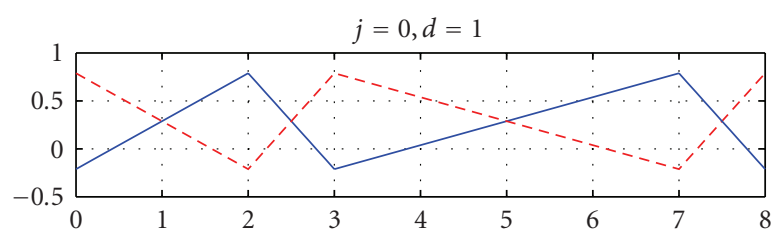

(a)

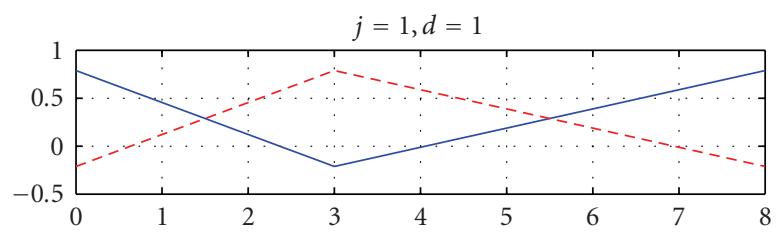

(b)

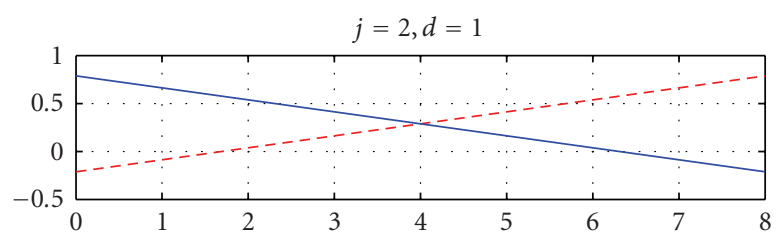

(c)

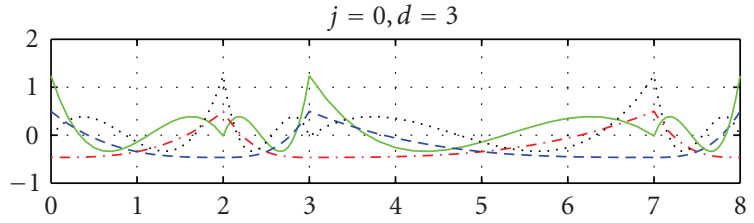

(a)

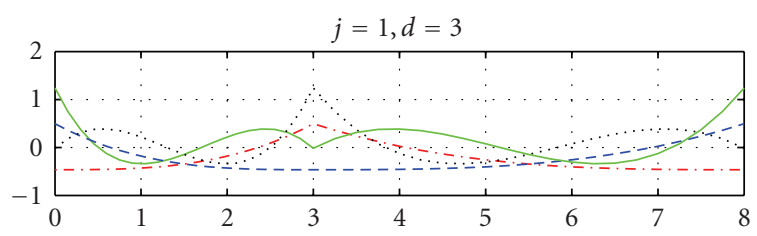

(b)

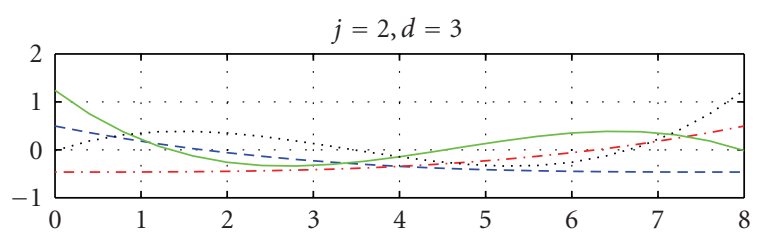

(c)

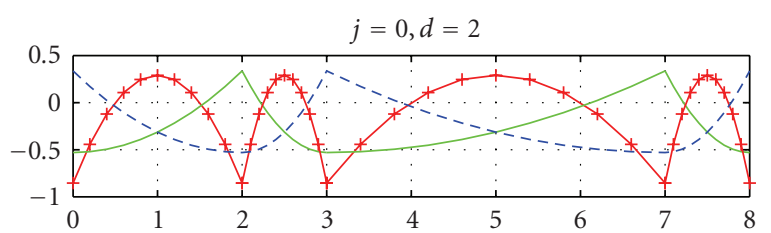

(a)

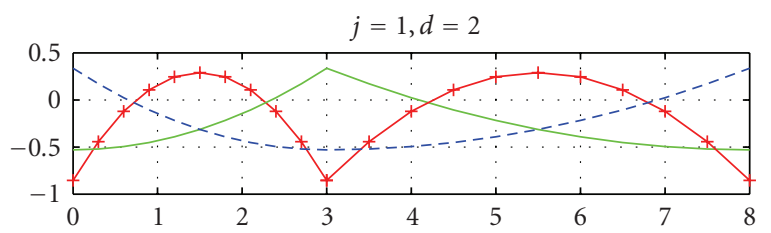

(b)

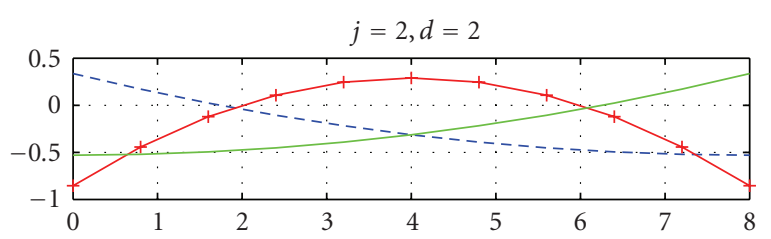

(c)

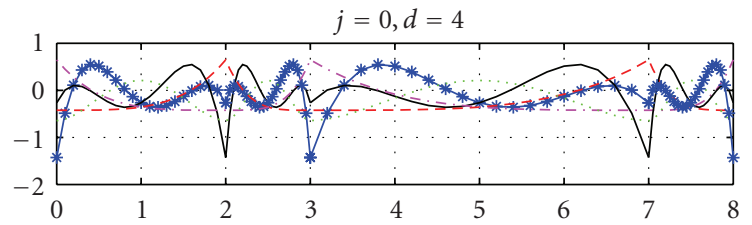

(a)

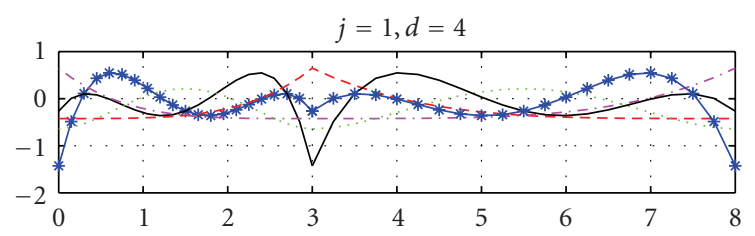

(b)

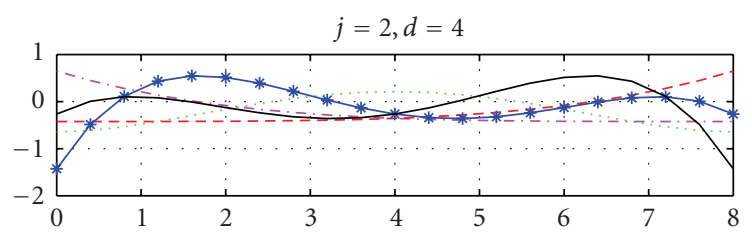

(c)

Figure 5: Orthonormal scaling spline bases at resolution levels $j=0,1,2$ from top to bottom $d=1$ (top left graphs), $d=2$ (top right graphs) $d=3$ (bottom left graphs), and $d=4$ (bottom right graphs) on the finest sequence $S_{0}=[0,2,3,7,8]$. in

(ii) the two symmetrical functions given by (17) resulting

$$
\begin{aligned}
& \left\langle b_{k, I_{j, i}}^{d}(t), b_{d-k, I_{j, i}}^{d}(t)\right\rangle=\delta_{k(d-k)} \\
& \quad \text { for } k=1, \ldots,\left\lfloor\frac{d-1}{2}\right\rfloor \quad \forall i, j \in \mathbb{N},
\end{aligned}
$$

where $\langle\cdot, \cdot\rangle$ represents the inner product.

It is easy to prove that conditions (19) impose the subsequent relation $s_{0, I_{j, i}}^{d}=s_{d, I_{j, i}}^{d}$. Moreover, these values are independent of the intervals leading therefore to the following equality $s_{0}^{d}=s_{d}^{d}$.

The resolution of the system of equations provided by the second conditions (20) shows that each shifting parameter associated to the corresponding transformed Bspline function is also the same constant value on each bounded interval. So, the shifting parameters are renamed as follows:

$$
s_{k, I_{j, i}}^{d}=s_{k}^{d} \quad \text { for } k=0, \ldots,\left\lfloor\frac{d}{2}\right\rfloor \quad \forall j \in \mathbb{N} .
$$




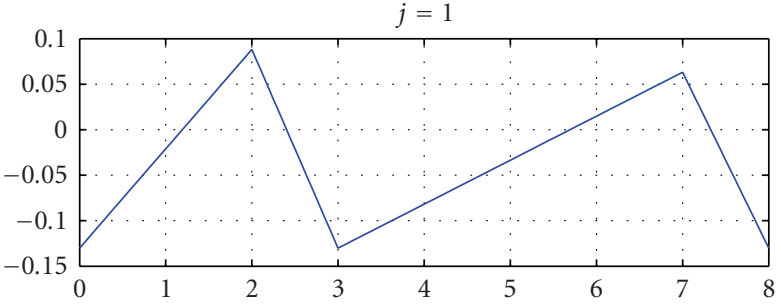

(a)

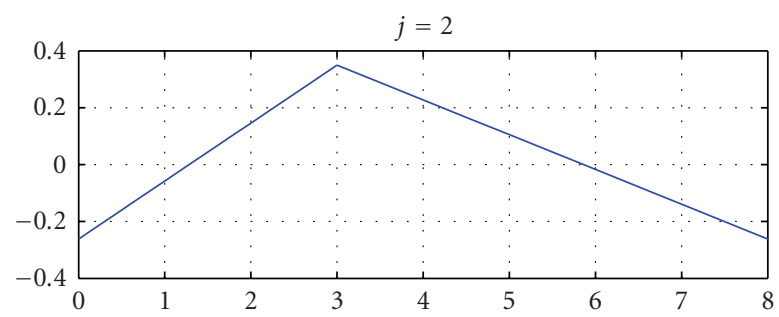

(b)

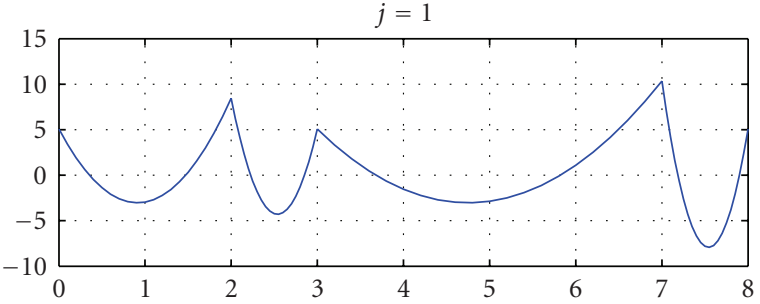

(a)

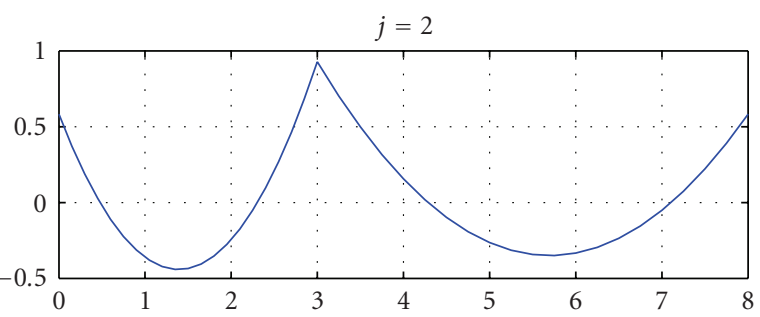

(b)

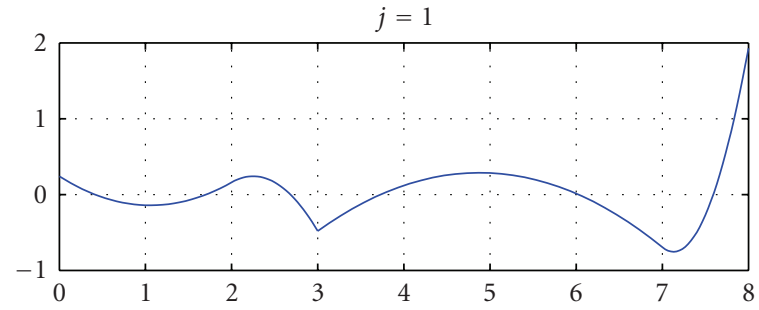

(a)

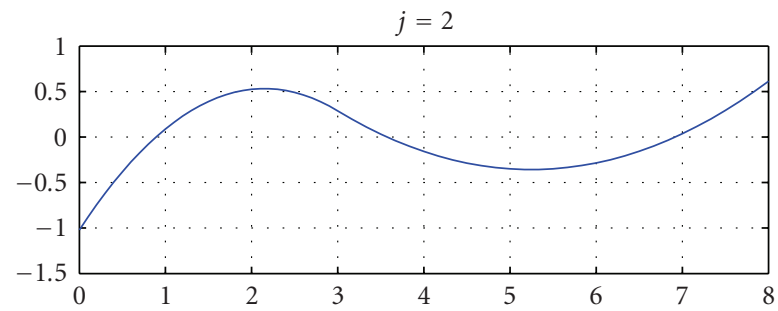

(b)

FIGURE 6: Normalized linear (left graphs) and quadratic (middle and right graphs) spline wavelet functions at $j=1,2$.

3.1.2. Weighting Parameters. For the second transformation, we introduce some weighting parameters $\left(m_{k, I_{j, i}}^{d}\right)$ as freedom parameters in the transformed B-spline functions given by (17) to control the continuity of the new orthogonal functions at the end-points of adjacent intervals (in step 4). The transformed spline functions are given as follows:

$$
\begin{aligned}
\underline{b}_{k, I_{j, i}}(t) & =s_{k}^{d}+m_{k, I_{j, i}}^{d} B_{k, I_{j, i}}^{d}(t) ; \\
\underline{b}_{d-k, I_{j, i}}(t) & =-s_{k}^{d}+m_{d-k, I_{j, i}}^{d} B_{d-k, I_{j, i}}^{d}(t) \\
& \text { for } k=1, \ldots,\left\lfloor\frac{(d-1)}{2}\right\rfloor .
\end{aligned}
$$

The particular transformed B-spline functions, given by (16) (i.e., the extreme and central (if $d$ is even) ones), do not require weighting parameters. Indeed they naturally ensure the continuity conditions with their neighbors. According to the particular values of the B-spline functions at the endpoints of their definition domain (see Figure 1), the functions $b_{0, I_{j, i}}^{d}(t), b_{d, I_{j, i}}^{d}(t)$ and $b_{d / 2, I_{j, i}}^{d}(t)$ (if $d$ is even), evaluated at the end-points of two consecutive intervals $I_{j, i}$ and $I_{j, i+1}$ are given by the following relations:

$$
\begin{array}{r}
b_{0, I_{j, i}}^{d}\left(t_{2^{j} i}\right)=b_{d, I_{j, i}}^{d}\left(t_{2^{j}(i+1)}\right)=b_{0, I_{j, i+1}}^{d}\left(t_{2^{j}(i+1)}\right)=s_{0}^{d}+1 ; \\
b_{0, I_{j, i}}^{d}\left(t_{2 j}(i+1)\right)=b_{d, I_{j, i}}^{d}\left(t_{2 j_{i}}\right)=b_{d, I_{j, i+1}}^{d}\left(t_{2^{j}(i+1)}\right)=s_{0}^{d}, \\
b_{d / 2, I_{j, i}}^{d}\left(t_{2^{j} i}\right)=b_{d / 2, I_{j, i}}^{d}\left(t_{2^{j}}(i+1)\right)=b_{d / 2, I_{j, i+1}}^{d}\left(t_{2^{j}}(i+1)\right)=s_{d / 2}^{d} \\
\text { if } d \text { is even } \quad \forall i, j \in \mathbb{N} .
\end{array}
$$

From these equations, we deduce that the continuity of the functions at common end-points of adjacent intervals is naturally satisfied if we swap between the functions $b_{0, I_{j, i}}^{d}(t)$ 


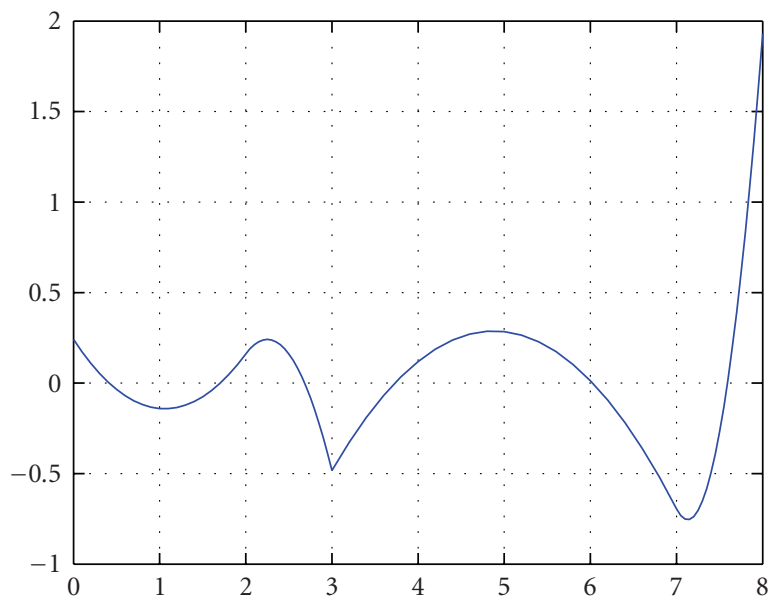

(a)

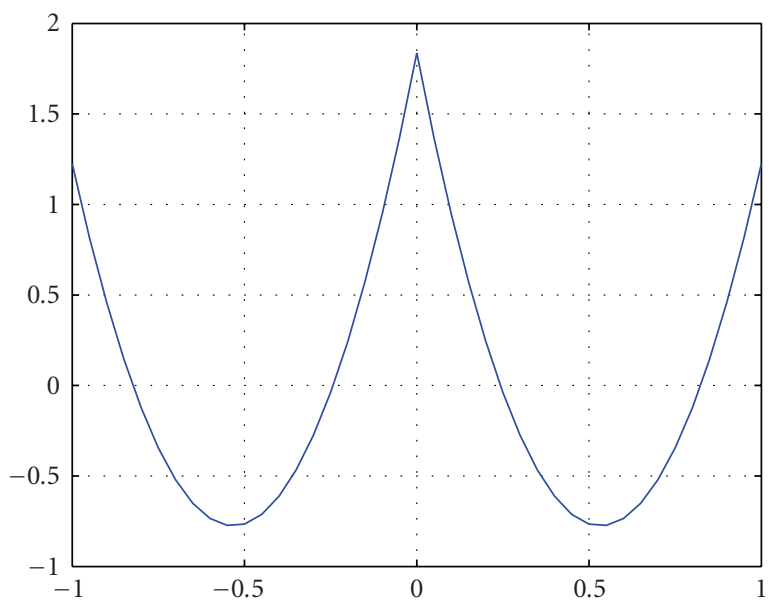

(c)

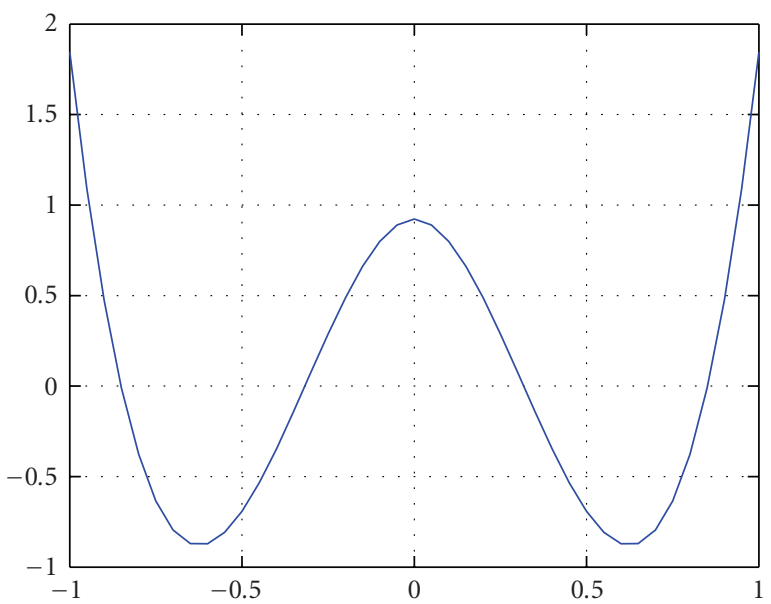

(b)

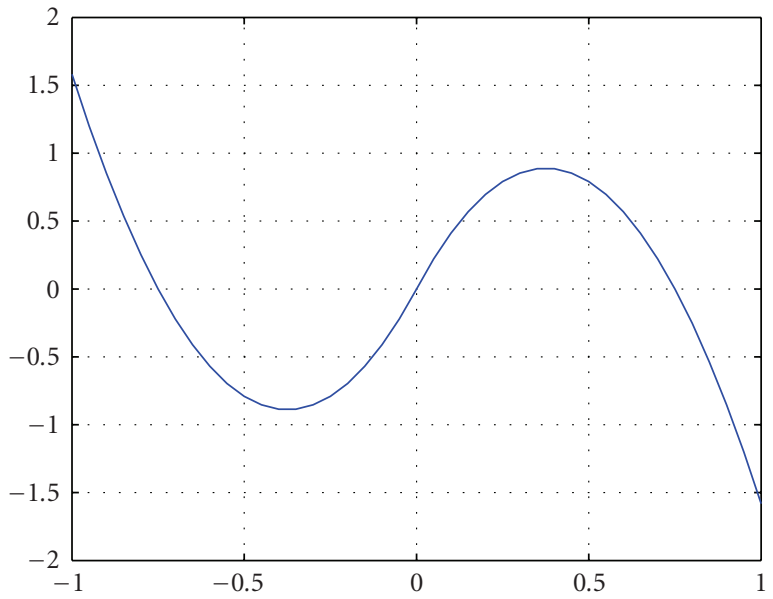

(d)

Figure 7: Normalized quadratic spline wavelet functions at resolution level $j=1$ satisfying the following conditions: (i) regularity $C^{0}$ with six vanishing moments (a); (ii) regularity $C^{1}$ in the interval $[-1,1]$ with four vanishing moments (b); (iii) regularity $C^{0}$ in the interval with four vanishing moments (c); (iv) regularity $C^{1}$ in the interval $[-1,1]$ with three vanishing moments (d).

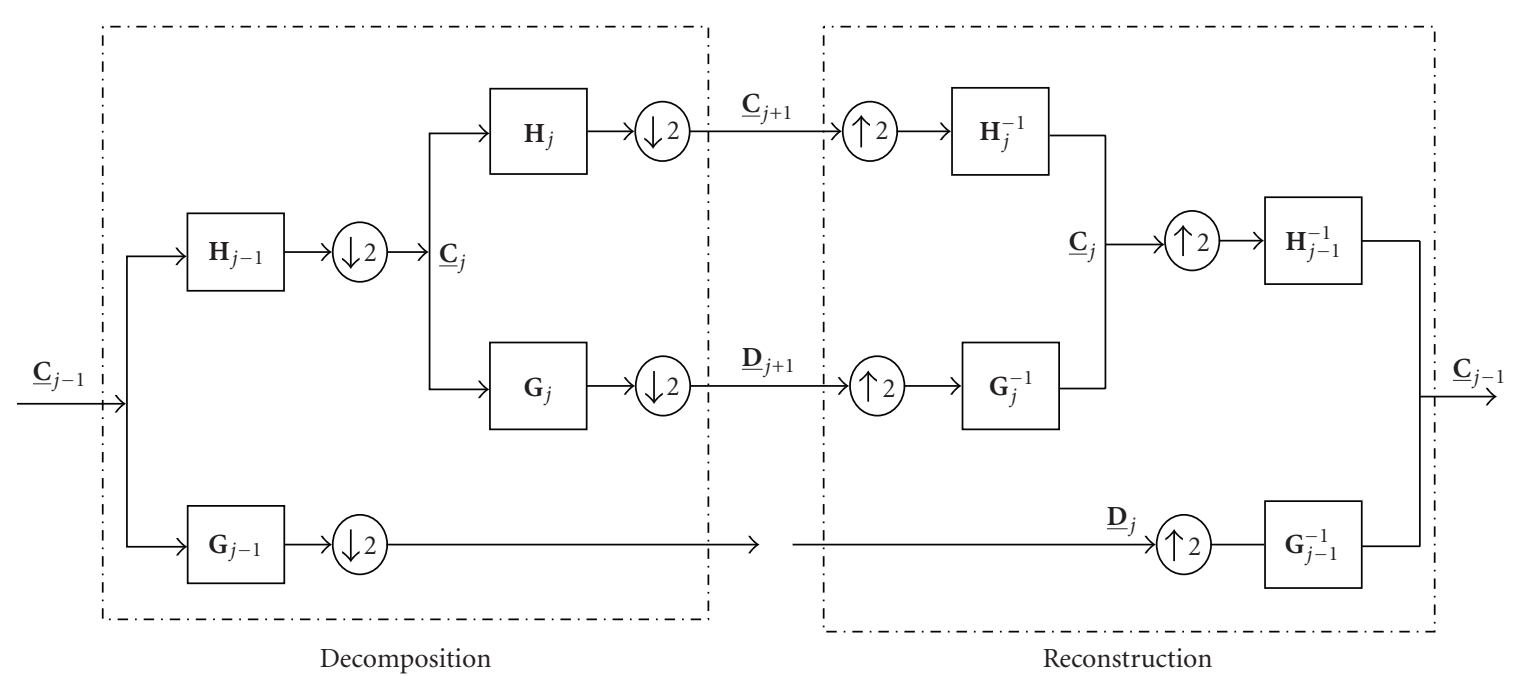

FIGURE 8: Multiresolution analysis using piecewise polynomial scaling and wavelet bases. 


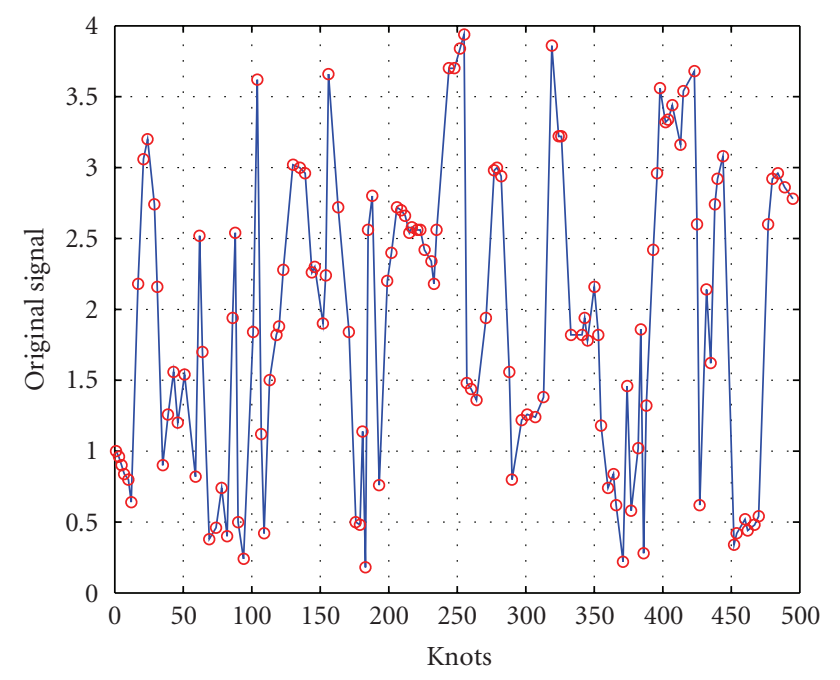

FIGURE 9: Original discrete signal irregularly spaced "o".

and $b_{d, I_{j, i}}^{d}(t)$ and from one interval to another. As for the function $b_{d / 2, I_{j, i}}^{d}(t)$ (if $d$ is even), it is clear that the continuity is naturally ensured on consecutive intervals. The first two or three (according to the parity of $d$ ) elements of the orthogonal basis (see (16)) are now renamed for writing convenience reasons as follows:

$$
\begin{gathered}
\underline{b}_{0, I_{j, i}}^{d}(t)=b_{0, I_{j, i}}^{d}(t) ; \quad \underline{b}_{d / 2, I_{j, i}}^{d}(t)=b_{d / 2, I_{j, i}}^{d}(t) ; \\
\underline{b}_{d, I_{j, i}}^{d}(t)=b_{d, I_{j, i}}^{d}(t) \quad \text { if } d \text { is even, } \quad \forall i, j \in \mathbb{N} \\
\underline{b}_{0, I_{j, i}}^{d}(t)=b_{0, I_{j, i}}^{d}(t) ; \quad \underline{b}_{d, I_{j, i}}^{d}(t)=b_{d, I_{j, i}}^{d}(t) \\
\text { if } d \text { is odd } \quad \forall i, j \in \mathbb{N} .
\end{gathered}
$$

3.2. Second Step: Global Orthogonalization. The second step takes care of the other orthogonality conditions. We use the classical Gram Schmidt algorithm to supplement the orthogonalization of the piecewise polynomial basis on each bounded interval. The extreme $\left(\underline{b}_{0, I_{j, i}}^{d}(t), \underline{b}_{d, I_{j, i}}^{d}(t)\right)$ and or the central $\left(\underline{b}_{d / 2, I_{j, i}}^{d}(t)\right)$ functions according to the parity of $d$, already satisfying the orthogonal conditions are chosen as reference components for the Gram Schmidt orthogonalization algorithm. Using the remaining parameterized functions given by (22), the Gram Schmidt method thus complements the construction of the orthogonal scaling basis in a traditional way. The resulting functions are denoted $\underline{b}_{k, I_{j, i}}^{d}(t)$. They are obviously orthogonal and parameterized by the weighting factors $m_{k, I_{j, i}}^{d}$ introduced earlier which are computed in the following step.

3.3. Third Step: Continuity between Adjacent Intervals. Step 3 is interested in the continuity of other functions of the orthogonal basis $\left(\underline{b}_{k, I_{j, i}}^{d}(t)\right.$ for $\left.k=1, \ldots,\lfloor(d-1) / 2)\right\rfloor$ than those given by (24). The weighting parameters, introduced in step 1 , are computed in order to guarantee the continuity
TABLE 1: Shifting and weighting parameters

\begin{tabular}{lcc}
\hline$d$ & Shifting parameters & Weighting parameters \\
\hline 1 & $s_{0}^{1}=-0.78$ & not required \\
2 & $s_{0}^{2}=-0.612, s_{0}^{2}=-0.373$ & not required \\
3 & $s_{0}^{3}=-0.485, s_{0}^{3}=-0.253$ & $m_{1}^{3}=1.925, m_{2}^{3}=5.07$ \\
$4 s_{0}^{4}=-0.395, s_{0}^{4}=-0.282, m_{0}^{4}=-0.159$ & $m_{1}^{4}=0.21, m_{2}^{4}=0.594$ \\
\hline
\end{tabular}

of each function $\underline{b}_{k, I_{j, i}}^{d}(t)$ at the common end-points of adjacent intervals. For this purpose, we impose the following conditions:

$$
\begin{array}{r}
\underline{b}_{k, I_{j, i}}^{d}\left(t_{2^{j} i}\right)=\underline{b}_{d-k, I_{j, i+1}}^{d}\left(t_{2^{j}(i+1)}\right) \\
\text { for } k=1, \ldots,\left\lfloor\frac{(d-1)}{2}\right\rfloor \quad \forall i, j \in \mathbb{N} .
\end{array}
$$

For a given spline function degree, the resolution of the system of equations provided by (25) shows that the weighting parameters are independent of the intervals whatever the resolution level $j$, leading to the following relation:

$$
m_{k, I_{j, i}}^{d}=m_{k}^{d} \quad \text { for } k=1, \ldots,\left\lfloor\frac{(d-1)}{2}\right\rfloor \quad \forall i, j \in \mathbb{N} .
$$

Table 1 provides the shifting and weighting parameters retained for the orthogonal piecewise polynomial scaling basis for different spline function degrees $d=1,2,3,4$.

3.4. Fourth Step: Normalization Factors. The main objective of the normalization step is to conserve the global finite energy (in $L^{2}(\mathbb{R})$ norm) of the original signal at each resolution level. In order to preserve the continuity of the scaling functions $\left(\underline{b}_{k, I_{i, i}}^{d}\right.$, in step 3 ), we propose to replace the local factor normalizations performed on each bounded interval with a global factor normalization $N_{j, k}^{d}$, computed on the global sequence $S_{j}=\bigcup_{i=0}^{N-1} I_{j, i}$ at each resolution level $j$, as follows:

$$
\begin{gathered}
\underline{\varphi}_{j, 0}^{d}(t)=\frac{\left(\sum_{i=0}^{N / 2} \underline{b}_{0, I_{j, 2 i}}(t)+\sum_{i=0}^{N / 2} \underline{b}_{0, I_{j, 2 i+1}}(t)\right)}{N_{j, 0}^{d}}, \\
\underline{\varphi}_{j, d / 2}^{d}(t)=\frac{\left(\sum_{i=0}^{N / 2} \underline{b}_{d / 2, I_{j, 2 i}}(t)+\sum_{i=0}^{N / 2} \underline{b}_{d / 2, I_{j, 2 i+1}}(t)\right)}{N_{j, d / 2}^{d}}, \\
\underline{\varphi}_{j, k}^{d}(t)=\frac{\left(\sum_{i=0}^{N / 2} \underline{b}_{k, I_{j, 2 i}}(t)+\sum_{i=0}^{N / 2} \underline{b}_{d-k, I_{j, 2 i+1}}(t)\right)}{N_{j, k}^{d}}, \\
\underline{\varphi}_{j, d-k}^{d}(t)=\frac{\left(\sum_{i=0}^{N / 2} \underline{b}_{d-k, I_{j, 2 i}}(t)+\sum_{i=0}^{N / 2} \underline{b}_{k, I_{j, 2 i+1}}(t)\right)}{N_{j, d-k}^{d}}, \\
\underline{\varphi}_{j, d}^{d}(t)=\frac{\left(\sum_{i=0}^{N / 2} \underline{b}_{d, I_{j, 2 i}}(t)+\sum_{i=0}^{N / 2} \underline{b}_{0, I_{j, 2 i+1}}(t)\right)}{N_{j, d}^{d}}, \\
\text { for } k=1, \ldots,\left\lfloor\frac{d-1}{2}\right\rfloor, \quad \forall j \in \mathbb{N},
\end{gathered}
$$

where $\underline{\varphi}_{j, k}^{d}(t)$ is the $k$ th global normalized piecewise polynomial scaling function at resolution level $j$. The basic 


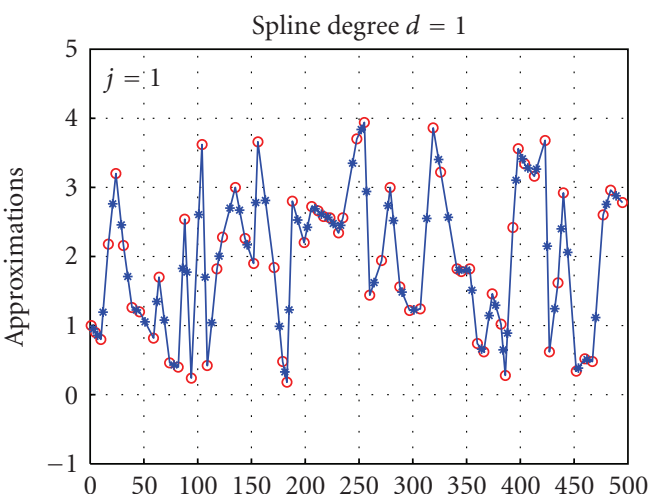

(a)

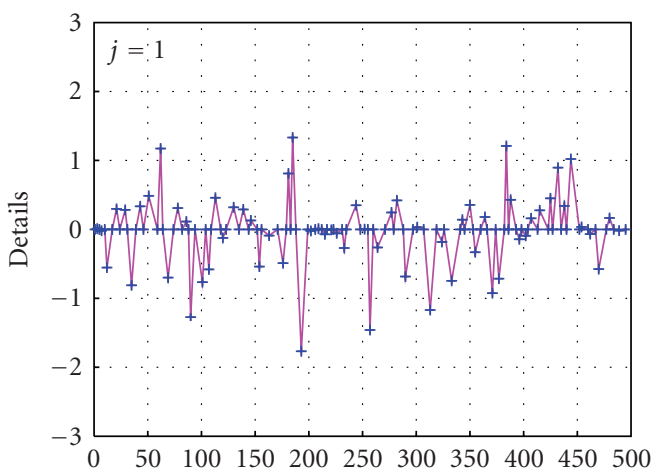

(c)

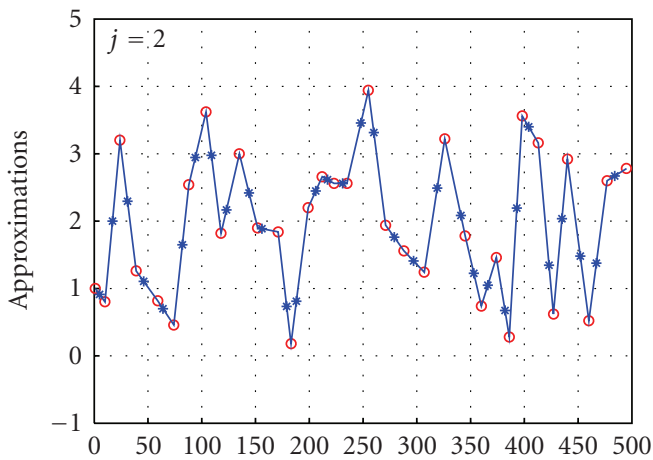

(e)

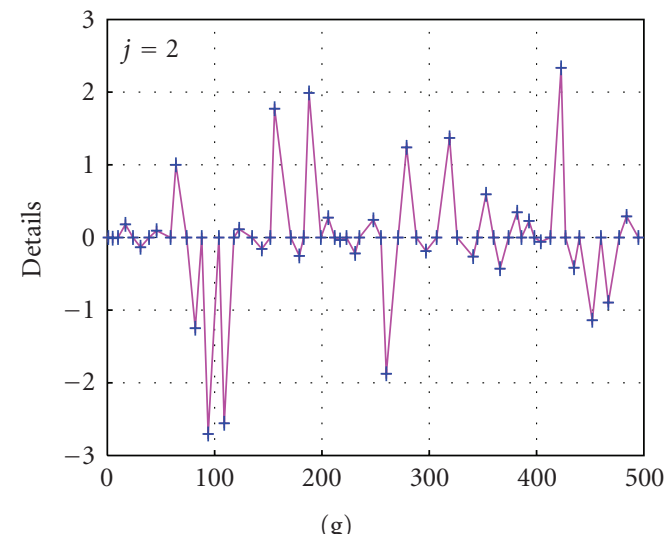

(g)

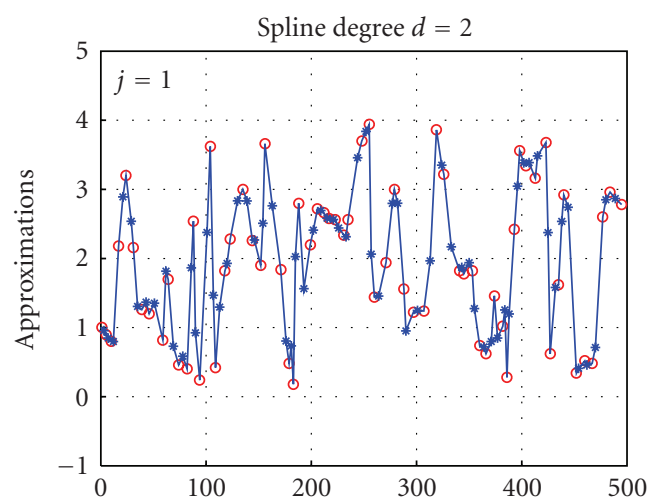

(b)

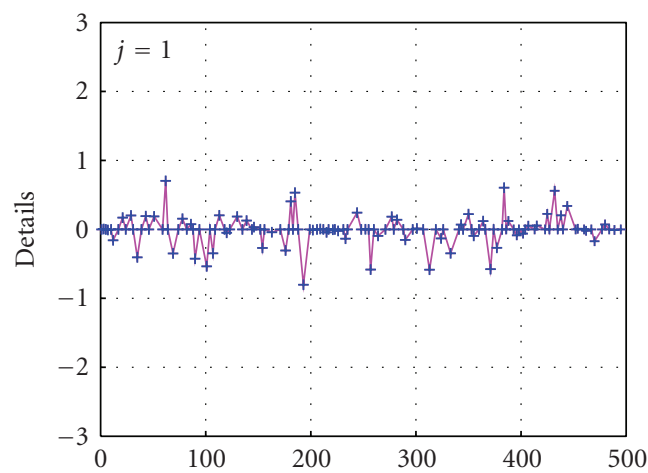

(d)

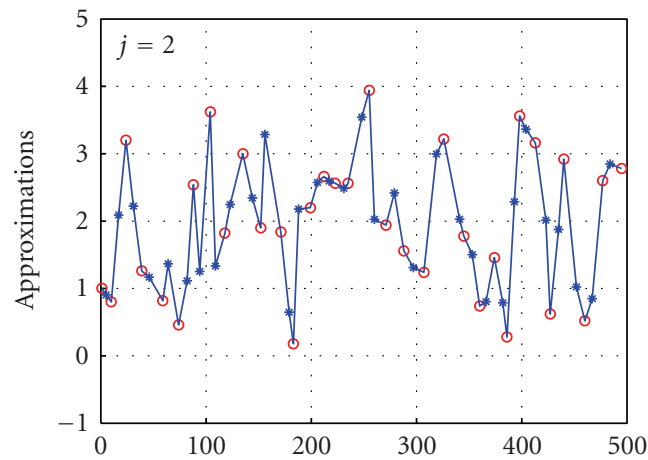

(f)

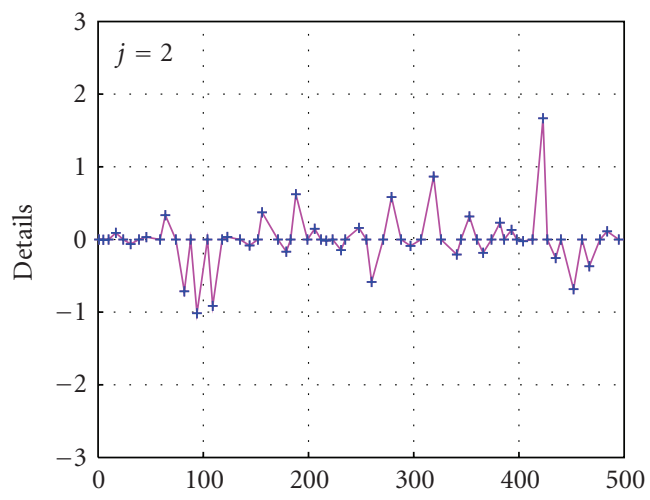

(h)

Figure 10: Orthogonal decomposition; “*” approximated signals, “o” irregularly discrete samples, “+” residual signals at respective resolution levels $j=1,2$ from linear and quadratic orthonormal piecewise polynomial scaling and wavelet bases. 
piecewise polynomial scaling space, whatever the degree of the spline function, is represented as

$$
\mathbb{V}_{0}^{d}=\left\{f: f(t)=\sum_{k=0}^{d} c_{k} \underline{\varphi}_{0, k}^{d}(t) \text { for } t \in S_{0} \quad \forall c_{k} \in l_{2}\right\}
$$

For convenience, the normalized basis functions are renamed as $\underline{B}_{k, I_{j, i}}^{d}(t)=\underline{b}_{k, I_{j, i}}^{d}(t) / n_{j, k}$ where the new orthonormalization factors $n_{j, k}$ are introduced according to the parity index $i$ of the bounded interval $I_{j, i}$ as follows:

$$
\begin{aligned}
& \text { if } i \text { is even: } n_{j, k}^{i}=N_{j, k} \text {; } \\
& \text { if } i \text { is odd: } n_{j, 0}^{i}=N_{j, d} ; n_{j, k}^{i}=N_{j, d-k}, n_{j, d-k}^{i}=N_{j, k} \text { and } \\
& n_{j, d}^{i}=N_{j, 0} \text { for } k=1, \ldots,\lfloor(d-1) / 2\rfloor .
\end{aligned}
$$

The orthonormal piecewise polynomial scaling basis is thus represented by

$$
\begin{aligned}
\underline{\varphi}_{j, 0}^{d}(t) & =\sum_{i=0}^{N / 2} \underline{B}_{0, I_{j, 2 i}}(t)+\sum_{i=0}^{N / 2} \underline{B}_{0, I_{j, 2 i+1}}(t), \\
\underline{\varphi}_{j, d / 2}^{d}(t)= & \sum_{i=0}^{N / 2} \underline{B}_{d / 2, I_{j, 2 i}}(t)+\sum_{i=0}^{N / 2} \underline{B}_{d / 2, I_{j, 2 i+1}}(t), \\
\underline{\varphi}_{j, k}^{d}(t)= & \sum_{i=0}^{N / 2} \underline{B}_{k, I_{j, 2 i}}(t)+\sum_{i=0}^{N / 2} \underline{B}_{d-k, I_{j, 2 i+1}}(t), \\
\underline{\varphi}_{j, d-k}^{d}(t) & =\sum_{i=0}^{N / 2} \underline{B}_{d-k, I_{j, 2 i}}(t)+\sum_{i=0}^{N / 2} \underline{B}_{k, I_{j, 2 i+1}}(t), \\
\underline{\varphi}_{j, d}^{d}(t) & =\sum_{i=0}^{N / 2} \underline{B}_{d, I_{j, 2 i}}(t)+\sum_{i=0}^{N / 2} \underline{B}_{0, I_{j, 2 i+1}}(t), \\
\text { for } k & =1, \ldots,\left\lfloor\frac{d-1}{2}\right\rfloor, \quad \forall j \in \mathbb{N} .
\end{aligned}
$$

Based on this new basis orthonormalization procedure, Figure 5 presents four examples $(d=1,2,3,4)$ of the orthonormal spline scaling bases of the respective spaces $\mathbb{V}_{0}^{d}$, $\mathbb{V}_{1}^{d}$ and $\mathbb{V}_{2}^{d}$ for the shifting and weighting parameters given in Table 1. The layouts are based on this finest knot sequence $S_{0}=\left[\tau_{0}^{d+1}=0, \tau_{1}^{d+1}=2, \tau_{2}^{d+1}=3, \tau_{3}^{d+1}=7, \tau_{4}^{d+1}=8\right]$. The top right graphs of Figure 5 present the continuous quadratic piecewise polynomial scaling functions. The dashed lines, on each resolution levels (graphs (a), (b), (c)), correspond to the function $\underline{\varphi}_{j, 1}^{2}(t)$. The solid lines are concerned with the function $\underline{\varphi}_{j, 1}^{2}(t)$ at each resolution level. Finally, the marked lines $(+)$ represent the function $\underline{\varphi}_{j, 0}^{2}(t)$ at each resolution level. The proposed scaling functions are continuous everywhere. The regularity of the scaling functions is of order zero at all joining intervals. This is inherent to the multiplicity which has been imposed on each knot of the considered sequence (see (5)).

\section{Piecewise Polynomial Wavelet Basis Using New Orthonormalization Procedure}

The following section is devoted to a unified orthonormal piecewise polynomial wavelet basis construction of the detail subspace whatever the degree of the spline function. Due to the multiplicity of the knots, we are going to show in the following pages that one can work with a single wavelet. Moreover, the continuity of the wavelet function being ensured again by relaxing the traditional normalization procedure (i.e., going to a normalization on each interval to a normalization on the global sequence).

4.1. Piecewise Polynomial Wavelet Subspace Dimension. Let us consider two consecutive intervals $I_{j-1, i} \cup I_{j-1, i+1}$ at resolution level $j-1$. As seen in Section 2 , the scaling and wavelet subspaces are linked by (14). The piecewise polynomial scaling subspace $\mathbb{V}_{0}^{d}\left[I_{0, i}\right] \cup \mathbb{V}_{0}^{d}\left[I_{0, i+1}\right]$ contains $\mathbb{W}_{1}^{d}\left[I_{1, i}\right]$. Therefore, any wavelet function $\psi_{k, I_{1, i}}^{d}(t) \in \mathbb{W}_{1}^{d}\left[I_{1, i}\right]$ defined on the bounded interval $I_{1, i}$ can be decomposed using the orthonormal basis of the scaling subspace $\mathbb{V}_{0}^{d}\left[I_{0, i}\right] \cup \mathbb{V}_{0}^{d}\left[I_{0, i+1}\right]$ as follows:

$$
\psi_{k, I_{1, i}}^{d}(t)=\sum_{m=2 i}^{2 i+1} \sum_{n=0}^{d} g_{1, k}^{m, n} \underline{B}_{n, I_{0, i}}^{d}(t) \quad \forall i, k \in \mathbb{N} .
$$

This wavelet function is parameterized by $2(d+1)$ coefficients, denoted $\left\{g_{1, k}^{m, n}\right\}$, which must be computed.

As the multiplicity $\mu$ of the knot to remove $\left(\tau_{2^{j-1}(i+1)}^{d+1}\right)$ decreases, the dimension of the piecewise polynomial scaling subspace, restricted to any interval $I_{j-1, i} \bigcup I_{j-1, i+1}$, decreases progressively leading to the nested scaling subspaces:

$$
\begin{aligned}
\mathbb{V}_{j-1}^{d}[ & \left.\tau_{2^{j-1} i}^{d+1}, \tau_{2^{j-1}(i+1)}^{d+1}\right] \bigcup \mathbb{V}_{j-1}^{d}\left[\tau_{2^{j-1}(i+1)}^{d+1}, \tau_{2^{j-1}(i+2)}^{d+1}\right] \\
& \supset \mathbb{V}_{j-1}^{d}\left[\tau_{2^{j-1} i}^{d+1}, \tau_{2^{j-1}(i+1)}^{d}, \tau_{2^{j-1}(i+2)}^{d+1}\right] \\
& \supset \cdots \supset \mathbb{V}_{j-1}^{d}\left[\tau_{2^{j-1} i}^{d+1}, \tau_{2^{j-1}(i+1)}^{1}, \tau_{2^{j-1}(i+2)}^{d+1}\right] \\
& \supset \mathbb{V}_{j-1}^{d}\left[\tau_{2^{j-1} i}^{d+1}, \tau_{2^{j-1}(i+2)}^{d+1}\right] \quad \forall i \in \mathbb{N}, j \geq 1 .
\end{aligned}
$$

According to (14), the piecewise polynomial wavelet subspace dimension, restricted to $I_{j, i}$, is deduced as follows:

$$
\begin{aligned}
\operatorname{dim}\left(\mathbb{W}_{j}^{d}\left[I_{j, i}\right]\right)= & \operatorname{dim}\left(\mathbb{V}_{j-1}^{d}\left[I_{j-1, i}\right] \bigcup \mathbb{V}_{j-1}^{d}\left[I_{j-1, i+1}\right]\right) \\
& -\operatorname{dim}\left(\mathbb{V}_{j}^{d}\left[I_{j, i}\right]\right)
\end{aligned}
$$

where the dimension of the scaling subspace $\left(\operatorname{dim}\left(\mathbb{V}_{j}^{d}\left[\tau_{2^{j} j}^{d+1}\right.\right.\right.$, $\left.\left.\tau_{2^{j}(i+1)}^{d+1}\right]\right)$ at resolution level $j$ is equal to $d+1$. According to 
(31), the dimension of the scaling subspace at resolution level $j-1$ is given as follows:

$$
\begin{aligned}
\operatorname{dim}\left(\mathbb{V}_{j-1}^{d}\left[\tau_{2^{j-1} i}^{d+1}, \tau_{2^{j-1}(i+1)}^{1}, \tau_{2^{j-1}(i+2)}^{d+1}\right]\right) \\
\leq \operatorname{dim}\left(\mathbb{V}_{j-1}^{d}\left[I_{j-1, i}\right] \bigcup \mathbb{V}_{j-1}^{d}\left[I_{j-1, i+1}\right]\right) \\
\leq \operatorname{dim}\left(\mathbb { V } _ { j - 1 } ^ { d } \left[\tau_{2^{j-1}(i+1)}^{d+1}, \tau_{2^{j-1}(i+2)}^{d+1}[\bigcup\right.\right. \\
\left.\mathbb{V}_{j-1}^{d}\left[\tau_{2^{j-1}(i+1)}^{d+1}, \tau_{2^{j-1}(i+2)}^{d+1}\right]\right) \quad \forall i \in \mathbb{N}, j \geq 1 .
\end{aligned}
$$

Then it translates into

$$
\begin{aligned}
d+2 & \leq \operatorname{dim}\left(\mathbb{V}_{j-1}^{d}\left[I_{j-1, i}\right] \bigcup \mathbb{V}_{j-1}^{d}\left[I_{j-1, i+1}\right]\right) \\
& \leq 2(d+1) \quad \forall i \in \mathbb{N}, \forall j \geq 1 .
\end{aligned}
$$

According to (32) and (34), we deduce that the dimension of the wavelet subspace, restricted to $I_{j, i}$, lies between one and $d+1$ :

$$
1 \leq \operatorname{dim}\left(\mathbb{W}_{j}^{d}\left[I_{j, i}\right]\right) \leq d+1 \quad \forall i \in \mathbb{N}, \forall j \geq 1 .
$$

This last equation means that we have the possibility of choosing several wavelet bases of various dimensions. Remember that the wavelet function $\psi_{k, I_{1, i}}^{d}(t)$ is parameterized by $2(d+1)$ coefficients $\left\{g_{1, k}^{m, n}\right\}$ (see $\left.(30)\right)$. In order to minimize the computational cost due to these coefficients, the smallest subspace dimension is retained. In theory, this choice requires to process the scaling subspace of dimension $d+2$ at resolution level $j-1$. However, it is easy to note that the approximated signal at the knot $\tau_{2^{j-1}(i+1)}^{\mu}$ (with $1 \leq$ $\mu \leq d+1)$ in any piecewise polynomial scaling subspaces at resolution level $j-1$ is the same value due to the chosen interpolation method used in this paper. Hence the quality of the detail signal at the knot $\tau_{2^{j-1}(i+1)}^{\mu}$ (with $1 \leq \mu \leq d+1$ ) is not affected by any particular selected scaling subspace dimension.

For computational simplicity, we choose the scaling subspaces of dimension $2(d+1)$ rather than $d+2$. The scaling functions belonging to the subspace of dimension $2(d+1)$ are easily computed since generalization formulas have been provided in [19]. Thus, at any resolution level $j$, equation (30) becomes

$$
\psi_{1, I_{j, i}}^{d}(t)=\sum_{m=2 i}^{2 i+1} \sum_{n=0}^{d} g_{j, 1}^{m, n} \underline{B}_{n, I_{j, i}}^{d}(t) \quad \forall i \in \mathbb{N}, \forall j \geq 1 .
$$

4.2. Computation of the Wavelet Coefficients Using Traditional Conditions. The wavelet function (see (36)) requires the computation of $2(d+1)$ unknown coefficients $\left\{g_{j, 1}^{m, n}\right\}$. For this purpose, we impose two sets of conditions. The first necessary set is directly related to the traditional multiresolution concept. On each interval of the knot sequence the wavelet subspace must be as follows:

(i) orthogonal to the scaling subspace, at any resolution level $(j \geq 1)$ resulting in

$$
\left\langle\psi_{1, I_{j, i}}^{d}(t), \underline{B}_{k, I_{j, i}}^{d}(t)\right\rangle=0 \quad \text { for } k=0, \ldots, \forall i \in \mathbb{N}, \forall j \geq 1
$$

(ii) orthogonal to the wavelet subspaces at all and cross scales, resulting in

$$
\left\langle\psi_{1, I_{j, i}}^{d}(t), \psi_{1, I_{k, p}}^{d}(t)\right\rangle=\alpha \delta_{j k} \delta_{i p} \quad \forall i, p \in \mathbb{N}, \forall j, k \geq 1,
$$

where $\alpha$ is a multiplicative constant; $\delta_{p q}$ is the Kronecker symbol which is equal to 1 if $p=q$ and to 0 if $p \neq q$.

Since conditions (37) and (38) do not fully determine $2(d+1)$ unknown coefficients $\left\{g_{j, 1}^{m, n}\right\}$, we introduce a second category of conditions. This category is concerned with additional useful conditions imposed on the wavelet so that the function satisfies the most significant desirable features often required for a "good" wavelet function (continuity, even differentiability, vanishing moments).

4.3. Computation of the Wavelet Coefficients Using Additional Conditions. These additional conditions have been studied extensively. We propose to establish condition priorities gathered in different classes depending on the degree of the spline function.

4.3.1. Continuity inside the Interval. In this category, we start with the first class of requirements ensuring the $C^{0}$ wavelet function regularity inside each interval $I_{j, i}$ at the particular knot $t_{2^{j-1}(i+1)}$ as follows:

$$
\begin{array}{r}
\sum_{n=0}^{d} g_{j, 1}^{2 i, n} \underline{B}_{n, I_{j-1, i}}^{d}\left(t_{2^{j-1}(i+1)}\right)=\sum_{n=0}^{d} g_{j, 1}^{2 i+1, n} \underline{B}_{n, I_{j-1, i}}^{d}\left(t_{2^{j-1}(i+1)}\right) \\
\forall i, k \in \mathbb{N}, \forall j \geq 1 .
\end{array}
$$

If the number of unknown coefficients $\left\{g_{1, k}^{m, n}\right\}$ for a given degree $d$ is less than the number of requirements, more conditions are necessary as described below.

4.3.2. Continuity at the Boundary Points of Consecutive Interval. The second class of conditions proposes to follow the $C^{0}$ regularity of the wavelet functions now on the global sequence $S_{j}$ at any resolution level $j$. In order to impose the continuity of the wavelets between different end-points of consecutive intervals, we suggest keeping one specific coefficient among the different coefficients $\left\{g_{j, 1}^{m, n}\right\}$ to guarantee the link between two consecutive intervals $I_{j, i}$ and $I_{j, i+1}$. The selected coefficient in the interval $I_{j, i+1}$ is then related to the selected coefficient in the preceding interval $I_{j, i}$ using the relationship deduced from the following equation:

$$
\psi_{1, I_{j, i}}^{d}\left(t_{2^{j}(i+1)}\right)=\psi_{1, I_{j, i+1}}^{d}\left(t_{2^{j}(i+1)}\right) \quad \forall i \in \mathbb{N}, \forall j \geq 1 .
$$

When going from one interval to the adjacent one, the selected coefficient (e.g., $g_{j, 1}^{m, n^{\prime}}$ with $0 \leq n^{\prime} \leq d$ ) in $I_{j, i+1}$ is then updated. If the number of unknown coefficients $\left\{g_{1, k}^{m, n}\right\}$ for a given degree $d$ is less than the number of requirements, more conditions are necessary as follows.

4.3.3. Controlling the Shape of the Wavelet Function on the Interval. The third class of conditions is primarily concerned 
with the shape of the wavelet on any bounded interval $I_{j, i}$. One can choose one among the four conditions to control the number of vanishing moments according to the desired shape of the wavelet function as given below:

$$
\begin{aligned}
& \psi_{1, I_{j, i}}^{d}\left(t_{2^{j}}\right) \\
& = \begin{cases} \pm \psi_{1, I_{j, i}}^{d}\left(t_{2^{j}(i+1)}\right) & \forall i \in \mathbb{N}, \forall j \geq 1, \\
\psi_{1, I_{j, i}}^{d}\left(t_{2^{j}}(i+1)\right) \pm \psi_{1, I_{j, i}}^{d}\left(t_{2^{j-1}(i+1)}\right) & \forall i \in \mathbb{N}, \forall j \geq 1 .\end{cases}
\end{aligned}
$$

Once again if the number of unknown coefficients $\left\{g_{1, k}^{m, n}\right\}$ for a given degree $d$ is less than the number of requirements, more conditions are necessary as described below.

4.3.4. Continuity of the First Derivative inside the Interval. The fourth class of conditions guarantees the $C^{1}$ wavelet function regularity inside any interval $I_{j, i}$. The continuity conditions of the first derivative of the wavelet function, at the particular knot $t_{2^{j-1}(i+1)}$ inside any interval $I_{j, i}$ in which the function belongs results in

$$
\begin{aligned}
& \frac{\partial \sum_{n=0}^{d} g_{j, 1}^{2 i, n} \underline{B}_{n, I_{j-1, i}}^{d}\left(t_{2^{j-1}(i+1)}\right)}{\partial t} \\
& =\frac{\partial \sum_{n=0}^{d} g_{j, 1}^{2 i+1, n} \underline{B}_{n, I_{j-1, i}}^{d}\left(t_{2^{j-1}(i+1)}\right)}{\partial t} \quad \forall i \in \mathbb{N}, \forall j \geq 1 .
\end{aligned}
$$

We keep increasing the list of conditions until reaching the maximum number of conditions, that is, $2(d+1)$. In this basis orthonormalization procedure we have chosen to ensure primarily the continuity of all achievable derivatives of the wavelet function at the particular point $t_{2^{j-1}(i+1)}$ inside the interval $I_{\mathrm{j}, i}$. Then we complete with the derivatives continuity conditions of the function at the end-points of $I_{j, i}$ if necessary.

4.3.5. Normalization on the Global Sequence. To preserve the $C^{0}$ regularity of the wavelet function as described previously, a global normalization factor is applied to the complete sequence $S_{j}$. Remember that one coefficient (e.g., $g_{j, 1}^{m, n^{\prime}}$ with $\left.0 \leq n^{\prime} \leq d\right)$ is used to ensure the $C^{0}$ wavelet regularity on $S_{j}$. The initial value of the coefficient $g_{j, 1}^{0, n^{\prime}}$ (in the first interval $\left.I_{j, 0}\right)$ is computed in such a way that the wavelet function is globally normalized on $S_{j}$ once for all.

4.3.6. Discussions. As seen above, a large family of piecewise polynomial wavelet basis can thus be constructed. But these wavelets are not all used to implement the multiresolution approach since they generate stability problems depending on a particular configuration of the knots in the sequence. Particular attention concerning the wavelet stability must be given when constructed. The stability problem occurs when the wavelet $\psi_{1, I_{j, i}}^{d}(t)$ evaluated at the knot $t_{2^{j-1}(i+1)}$ inside the interval $I_{j, i}$ or at the end-point $t_{2^{j}(i+1)}$ of the same interval is equal to zero. If the wavelet function is equal to zero at the point $t_{2 j(i+1)}$, the chosen parameter $g_{j, 1}^{m, n^{\prime}}$ is automatically equal to zero therefore leading to wavelet functions $\psi_{1, I_{i, i}}^{d}(t)$ which are null on each interval of the considered sequence (see (40)). To overcome the stability problems, the wavelet function must meet the following requirements:

$$
\psi_{1, I_{j-1, i}}^{d}(t) \neq 0, \quad \psi_{1, I_{j-1, i}}^{d}(t) \neq 0, \quad g_{j, 1}^{m, n} \neq 0 \quad \forall j \geq 1 .
$$

The wavelet, denoted by $\psi_{j}^{d}(t)$, of the detail subspace $\mathbb{W}_{j}^{d}$ is now deduced as follows:

$$
\psi_{j}^{d}(t)=\sum_{i=0}^{N-1} \psi_{1, I_{j, i}}^{d}(t) \quad \forall j \geq 1 .
$$

With this wavelet basis orthonormalization procedure, the wavelet regularity inside the interval is $C^{d-1}$ for $d>2$. Increasing the spline function degree allows us to ensure the wavelet derivatives continuity everywhere.

Figure 6 presents the linear (left graphs) and quadratic (middle and right graphs with different relationships at the boundary points) piecewise polynomial wavelet at two resolution levels $j=1$ (graph (a)) and $j=2$ (graph (b)) constructed on the previous finest initial knot sequence: $S_{0}=[0,2,3,7,8]$. The number of wavelet functions initially equal to two for $d=1$ and three for $d=2$, as described in Section 2.3, is reduced to only one function on each bounded interval. Moreover, the quadratic wavelet is continuous inside the bounded interval (regularity $C^{0}$ ) and at the end-point of adjacent intervals. It is possible to increase the regularity of the quadratic wavelet inside each bounded interval if the condition (41) is replaced by the continuity condition of the wavelet first derivatives at the point inside the considered interval. Examples are given by the second and fourth graphs of Figure 7 where the continuity of the quadratic wavelet first derivative at the point 0 is ensured. Rather than satisfying the condition given by (40), one can increase the number of vanishing moments of the wavelet such as illustrated by the first graph of Figure 7. However, conditions (41) are useful for decomposing any discret signal regularly sampled. Although the wavelet basis is orthogonal, the proposed wavelet becomes symmetric and compactly supported (see the graphs (b), (c) and (d) of Figure 7).

\section{Orthogonal Decomposition Algorithm}

This section is concerned with the orthogonal decomposition of a signal $y(t)$ represented by its discrete samples irregularly spaced $y\left(t_{i}\right)$. We assume that this signal lives in the basic spline space $V_{0}^{d}$. The approximation of the signal $y(t)$ at resolution level $j$, on each bounded interval $I_{j, i}$, is denoted $y_{I_{j, i}}(t)$. This signal belongs to the scaling space $V_{j}^{d}\left[I_{j, i}\right]$. As seen earlier, the piecewise polynomial scaling and wavelet subspaces satisfy the orthogonal property given by (14). Therefore, one can decompose any signal $y_{I_{j-1, i}}(t)+$ $y_{I_{j-1, i+1}}(t) \in V_{j-1}^{d}\left[I_{j-1, i}\right] \cup V_{j-1}^{d}\left[I_{j-1, i+1}\right]$, according to the relation

$$
y_{I_{j-1,2 i}}(t)+y_{I_{j-1,2 i+1}}(t)=y_{I_{j, i}}(t)+r_{I_{j, i}}(t) \quad \forall i \in \mathbb{N}, \forall j \geq 1,
$$


where $r_{I_{j, i}}(t)$ is the detail or residual signal at resolution level $j$, defined on the interval $I_{j, i}$. The residual signal belongs to the wavelet space $W_{j}^{d}\left[I_{j, i}\right]$. Since the signal $y_{I_{j, i}}(t)$ belongs to the scaling space $V_{j}^{d}$, one can decompose it using the orthonormal scaling basis of the corresponding space $V_{j}^{d}\left[I_{j, i}\right]$ as follows:

$$
y_{I_{j, i}}(t)=\sum_{k=0}^{d} c_{j, k}^{i} \underline{\varphi}_{k, I_{j, i}}^{d}(t) \quad \forall i \in \mathbb{N}, \forall j \geq 1
$$

For the same reasons, the residual signal $r_{I_{j, i}}(t)$ can be expressed using the wavelet basis of the space $W_{j}^{d}\left[I_{j, i}\right]$ as follows:

$$
r_{I_{j, i}}(t)=d_{j, 1}^{i} \psi_{1, I_{j, i}}^{d}(t) \quad \forall i \in \mathbb{N}, \forall j \geq 1 .
$$

Thus, the approximated signal $y_{I_{j-1, i}}(t) \in V_{j-1}^{d}\left[I_{j, i}\right]$ is given by the following relation:

$$
\begin{array}{r}
y_{I_{j-1,2 i}}(t)+y_{I_{j-1,2 i+1}}(t)=\sum_{k=0}^{d} c_{j, k}^{i} \underline{\varphi}_{\mathrm{k}, I_{j, i}}^{d}(t)+d_{j, 1}^{i} \psi_{1, I_{j, i}}^{d}(t) \\
\forall i \in \mathbb{N}, \forall j \geq 1,
\end{array}
$$

where the weighted coefficients $c_{j, k}^{i}$ (resp., $d_{j, 1}^{i}$ ) are given by the orthogonal projection of $y_{I_{j, i}}(t)$ (resp., $\left.r_{I_{j, i}}(t)\right)$ on the scaling subspace $V_{j}^{d}\left[I_{j, i}\right]$ (resp., $W_{j}^{d}\left[I_{j, i}\right]$ ).

Going from (48), the set of coefficients $c_{j, k}^{i}$ (level $j$ ) are closely related to coefficients $c_{j-1, k}^{i}$ (level $j-1$ ) by means of the coefficients set, denoted $h_{j, n}^{l, k}$, on each bounded interval $I_{j, i}$, as follows:

$$
\begin{aligned}
& \underline{\mathbf{c}}_{I_{j, i}}=\underline{\mathbf{H}}_{I_{j, i}} \mathbf{c}_{I_{j-1, i}} \quad \text { with } \\
& \underline{\mathbf{c}}_{I_{j-1, i}}=\left(\begin{array}{llllllll}
c_{j-1,0}^{2 i} & c_{j-1,1}^{2 i} & \cdots & c_{j-1, d}^{2 i} & c_{j-1,0}^{2 i+1} & c_{j-1,1}^{2 i+1} & \cdots & c_{j-1, d}^{2 i+1}
\end{array}\right)^{t}, \\
& \underline{\mathbf{c}}_{I_{j, i}}=\left(\begin{array}{llll}
c_{j-1,0}^{2 i} & c_{j-1,1}^{2 i} & \cdots & c_{j-1, d}^{2 i}
\end{array}\right)^{t} \quad \forall i \in \mathbb{N}, \forall j \geq 1 .
\end{aligned}
$$

Due to the basis orthonormalization procedure (see (29)), the decomposition matrix $\underline{\mathbf{H}}_{I_{j, i}}$ depends on the parity of the index $i$ corresponding to the interval $I_{j, i}$ in the sequence as follows:

$$
\begin{aligned}
& \text { If } i \text { is even then } \underline{\mathbf{H}}_{I_{j, i}}=\left(\begin{array}{cccccccc}
h_{j, 0}^{2 i, 0} & h_{j, 1}^{2 i, 1} & \cdots & h_{j, 0}^{2 i, d} & h_{j, 0}^{2 i+1,0} & h_{j, 0}^{2 i+1,1} & \cdots & h_{j, 0}^{2 i+1, d} \\
h_{j, 1}^{2 i, 0} & h_{j, 1}^{2 i, 1} & \cdots & h_{j, 1}^{2 i, d} & h_{j, 1}^{2 i+1,0} & h_{j, 1}^{2 i+1,1} & \cdots & h_{j, 1}^{2 i+1, d} \\
\vdots & \vdots & \vdots & \vdots & \vdots & \vdots & \vdots & \vdots \\
h_{j, d-1}^{2 i, 0} & h_{j, d-1}^{2 i, 1} & \cdots & h_{j, d-1}^{2 i, d} & h_{j, d-1}^{2 i, 0} & h_{j, d-1}^{2 i, 1} & \cdots & h_{j, d-1}^{2 i, d} \\
h_{j, d}^{2 i, 0} & h_{j, d}^{2 i, 1} & \cdots & h_{j, d}^{2 i, d} & h_{j, d}^{2 i, 0} & h_{j, d}^{2 i, 1} & \cdots & h_{j, d}^{2 i, d}
\end{array}\right) \quad \forall j \geq 1 \text {, } \\
& \text { If } i \text { is odd then } \underline{\mathbf{H}}_{I_{j, i}}=\left(\begin{array}{cccccccc}
h_{j, d}^{2 i, 0} & h_{j, d}^{2 i, 1} & \cdots & h_{j, d}^{2 i, d} & h_{j, d}^{2 i, 0} & h_{j, d}^{2 i, 1} & \cdots & h_{j, d}^{2 i, d} \\
h_{j, d-1}^{2,0} & h_{j, d-1}^{2 i, 1} & \cdots & h_{j, d-1}^{2,, d} & h_{j, d-1}^{2 i, 0} & h_{j, d-1}^{2 i, 1} & \cdots & h_{j, d-1}^{2 i, d} \\
\vdots & \vdots & \vdots & \vdots & \vdots & \vdots & \vdots & \vdots \\
h_{j, 1}^{2 i, 0} & h_{j, 1}^{2 i, 1} & \cdots & h_{j, 1}^{2 i, d} & h_{j, 1}^{2 i+1,0} & h_{j, 1}^{2 i+1,1} & \cdots & h_{j, 1}^{2 i+1, d} \\
h_{j, 0}^{2 i, 0} & h_{j, 1}^{2 i, 1} & \cdots & h_{j, d}^{2 i, d} & h_{j, 0}^{2 i+1,0} & h_{j, 0}^{2 i+1,1} & \cdots & h_{j, 0}^{2 i, 1, d}
\end{array}\right) \quad \forall j \geq 1 \text {. }
\end{aligned}
$$

The matrix dimensions are given below:

$$
\begin{gathered}
\operatorname{dim}\left(\underline{\mathbf{c}}_{I_{j, i}}\right)=(d+1) \times 1, \\
\operatorname{dim}\left(\underline{\mathbf{H}}_{I_{j, i}}\right)=(d+1) \times 2(d+1), \\
\operatorname{dim}\left(\underline{\mathbf{c}}_{I_{j-1, i}}\right)=2(d+1) \times 1 .
\end{gathered}
$$

The extension of (49) to the sequence $S_{j}$ is easily obtained and becomes

$$
\begin{aligned}
& \underline{\boldsymbol{c}}_{j}=\underline{\mathbf{H}}_{j} \underline{\mathbf{C}}_{j-1} \quad \text { with } \quad\left(\begin{array}{lll}
\underline{\mathbf{c}}_{I_{j, 0}} & \underline{\mathbf{c}}_{I_{j, 1}} & \cdots
\end{array}\right)^{t}, \\
& \underline{\mathbf{H}}_{j}=\left(\begin{array}{cccc}
\underline{\mathbf{H}}_{I_{j, 0}} & {[0]} & {[0]} & {[0]} \\
{[0]} & \underline{\mathbf{H}}_{I_{j, 1}} & {[0]} & {[0]} \\
\vdots & {[0]} & \ddots & {[0]} \\
{[0]} & {[0]} & {[0]} & \underline{\mathbf{H}}_{I, n}
\end{array}\right) .
\end{aligned}
$$


The computation of the detail coefficients at any resolution level $j$, on the interval $I_{j, i}$, are also given by the following relation:

$$
\begin{aligned}
\underline{\mathbf{d}}_{I_{j, i}}= & \underline{\mathbf{G}}_{I_{j, i}} \mathbf{c}_{I_{j-1, i}} \text { with } \\
\underline{\mathbf{c}}_{I_{j-1, i}} & =\left(\begin{array}{llllllll}
c_{j-1,0}^{2 i} & c_{j-1,1}^{2 i} & \cdots & c_{j-1, d}^{2 i} & c_{j-1,0}^{2 i+1} & c_{j-1,1}^{2 i+1} & \cdots & c_{j-1, d}^{2 i+1}
\end{array}\right)^{t}, \\
\underline{\mathbf{G}}_{I_{j, i}}= & \left(\begin{array}{cccccccc}
\hbar_{j, 0}^{2 i, 0} & \hbar_{j, 1}^{2 i, 1} & \cdots & \hbar_{j, 0}^{2 i, d} & \hbar_{j, 0}^{2 i+1,0} & \hbar_{j, 0}^{2 i+1,1} & \cdots & \hbar_{j, 0}^{2 i+1, d} \\
0 & 0 & \cdots & 0 & 0 & 0 & \cdots & 0 \\
\vdots & \vdots & \vdots & \vdots & \vdots & \vdots & \vdots & \vdots \\
0 & 0 & \cdots & 0 & 0 & 0 & \cdots & 0
\end{array}\right)
\end{aligned}
$$

The dimensions of the matrices are equivalent to those provided in (52). The main important difference between the previous basis orthonormalization procedure of the piecewise polynomial scaling and wavelet basis (see [21]) is that the matrix $\underline{\mathbf{d}}_{I_{j, i}}$ is reduced from $d+1$ values to only one value $d_{j, 1}^{i}$ (different from zero) on each bounded interval $I_{j, i}$. Moreover, the number of coefficients of the filter $\underline{\mathbf{G}}_{I_{j, i}}$, previously equal to $2(d+1)^{2}$ coefficients, is reduced to only $2(d+1)$ coefficients. Reducing therefore the multiresolution filter banks complexity. Equation (54), valid on each interval, is extended to the sequence $S_{j}$ as follows:

$$
\underline{\mathbf{D}}_{j}=\mathbf{G}_{j} \underline{\mathbf{C}}_{j} \quad \text { with } \quad \underline{\mathbf{D}}_{j}=\left(\begin{array}{lll}
\underline{\mathbf{d}}_{I_{j, 0}} & \underline{\mathbf{d}}_{I_{j, 1}} & \cdots
\end{array}\right)^{t} \quad \forall j \geq 1,
$$

where $\mathbf{G}_{j}$ is the extension of the matrix decomposition $\mathbf{G}_{I_{j, i}}$ to the sequence $S_{j}$ equivalent to $\mathbf{H}_{I_{j, i}}$. Since the decomposition is orthogonal, the reconstruction matrices are deduced from the decomposition matrices $\mathbf{H}_{j}$ and $\mathbf{G}_{j}$. These matrices are sparse, which allows efficient decomposition and reconstruction steps. The traditional filters bank are replaced by a set of filters $\left(\mathbf{G}_{I_{j, i}}, \mathbf{H}_{I_{j, i}}\right)$ which depend on the position of the knots in the sequence $S_{j}$. Figure 8 presents the global multiresolution scheme for two consecutive resolution levels.

\section{Implementation of the Multiresolution Approach}

This section addresses the implementation of the proposed multiresolution approach in the context of irregularly spaced data. The orthogonal decomposition, as seen earlier, processes approximation coefficients $\underline{\mathbf{C}}_{j}$ at each resolution level. Therefore an initialization step is required to find the first initial coefficients set $\underline{\mathbf{C}}_{0}$. The computation of these coefficients needs the manipulation of the continuous signal $y(t)$. However, the signal is represented only by its irregularly spaced samples $y\left(t_{i}\right)$ with $t_{i} \in S_{0}$. For this purpose, we assume that this signal belongs to the basic spline space $V_{0}^{d}$ spanned by the spline basis provided by (6) in Section 2 . We propose to interpolate the discrete signal, on each bounded interval $I_{0, i}$, using the nonuniform B-spline functions $\left\{B_{k, I_{0, i}}^{d}\right\}$ of the basic space $V_{0}^{d}\left[I_{0, i}\right]$ according to the relation

$$
y_{I_{0, i}}(t)=\sum_{k=0}^{d} a_{k, I_{0, i}} B_{k, I_{0, i}}^{d} \quad \forall i \in \mathbb{N},
$$

where the computation of the weighted coefficients $\left\{a_{k, I_{0, i}}\right\}$ has been extensively studied in previous works (see, e.g., [18]). Recall that the basic spline space $V_{0}^{d}$ is also represented by the orthonormal piecewise polynomial scaling basis $\left\{\underline{\varphi}_{k, I_{0, i}(t)}\right\}$. The orthogonal projection of the signal $y_{I_{0, i}}(t)$, on the basic spline space $V_{0}^{d}\left[I_{0, i}\right]$ allows the computation of the approximation coefficients $\underline{\mathrm{C}}_{0}$ as follows:

$$
c_{0, k}^{i}=\left\langle y_{I_{0, i}}(t), \underline{\varphi}_{k, I_{0, i}}^{d}\right\rangle \quad \text { for } k=0, \ldots, d, \forall i \in \mathbb{N} .
$$

The coefficients $\underline{\mathrm{C}}_{0}$ are thus provided by replacing $y_{I_{0, i}}(t)$ in the previous expression as follows:

$$
c_{0, k}^{i}=\sum_{l=0}^{d} a_{l, I_{0, i}}\left\langle B_{l, I_{0, i}}^{d}(t), \underline{\varphi}_{k, I_{0, i}}^{d}(t)\right\rangle \quad \text { for } k=0, \ldots, d, \forall i \in \mathbb{N} \text {. }
$$

The computation of the coefficients set $\underline{\mathrm{C}}_{0}$ given by (58) uses the B-spline coefficients values provided by Table 2 for $d=1$ and $d=2$ (see [18]). In the quadratic case, the coefficient $a_{l, I_{0, i}}$ requires the first derivative value of the signal $y_{I_{0, i}}(t)$ evaluated at the knot $t_{i}$ (see Table 2). Let us point out that the quality of the multiresolution approach depends closely on the computation method from which the first derivative $y_{I_{0, i}}^{\prime}(t)$ is evaluated. We propose to construct a quadratic polynomial which passes through three consecutive known samples $y_{I_{0, i}}\left(t_{i}\right), y_{I_{0, i}}\left(t_{i+1}\right)$, and $y_{I_{0, i}}\left(t_{i+2}\right)$. It is then easy to deduce the first derivative value taken at the knot $t_{i}$.

Figure 9 provides the original signal represented by its 129 samples irregularly spaced. This signal, randomly subsampled, corresponds to line 24 of the Lena image $(512 \times$ 512 pixels). In order to analyze the results, the magnitude of the original signal has been scaled by a factor of 50 . The retained samples are marked by the symbol "o." This signal is decomposed using both the orthonormal linear and quadratic piecewise polynomial scaling and wavelet bases developed in the preceding sections (see Figures 5 and 6). The graphs in the left column (resp., of the right column) of Figure 10 present the discrete approximated signals and residual signals using linear (resp., quadratic) spline scaling and wavelet bases for two resolution levels. The corresponding available samples at resolution $j$ are represented by the symbol "o," and the approximated signals use the symbol " $*$ " at each resolution level. The residual signals are represented by the symbol “+." A large number of the wavelet transform detail samples turn out to be very small in magnitude, as shown in Figure 10. One can clearly notice that increasing the degree of the piecewise polynomial scaling and wavelet function results in a smaller variance of the residual signal. The most interesting case corresponds to piecewise polynomial of degrees greater than 
TABLE 2: B-spline coefficients.

\begin{tabular}{cc}
\hline$d$ & $a_{0, I_{0, i}}$ \\
\hline 1 & $a_{0, I_{0, i}}=y_{I_{0, i}}\left(t_{i}\right), a_{1, I_{0, i}}=y_{I_{0, i}}\left(t_{i+1}\right)$ \\
& $a_{0, I_{0, i}}=y_{I_{0, i}}\left(t_{i}\right), a_{2, I_{0, i}}=y_{I_{0, i}}\left(t_{i+1}\right)$, \\
2 & $a_{1, I_{0, i}}=y_{I_{0, i}}\left(t_{i}\right)+y_{I_{0, i}}^{\prime}\left(t_{i}\right)\left(t_{i+1}-t_{i}\right) / 2$ \\
\hline
\end{tabular}

one (linear case). The linear case is a commonplace case. Indeed, we do not need to use the developed theory since one knows how to connect directly two consecutive points by a line. For degrees higher than one, it is necessary however to evaluate the derivatives of corresponding order of the signal. Moreover, as already stated, the better the approximation of these derivatives, the smaller the variance of the residual signal.

\section{Conclusion}

This paper explored the underlying mathematical framework of the one-dimensional multiresolution analysis based on the nonequally spaced samples environment. The study shows that the scaling and wavelet functions are not respectively given by dilating and translating one unique prototype function as in the traditional case. The specification of the multiresolution spaces involves the construction of the corresponding scaling and wavelet bases. As shown in previous work, the orthonormalization procedure has affected the regularity of the piecewise polynomial scaling and wavelet functions. This paper has proposed a new basis orthonormalization procedure which (i) reduces the number of the wavelets to a single one per interval (depending on the localization of the samples) and (ii) satisfies the continuity conditions of the scaling and wavelet functions on the considered knot sequence. Moreover the orthogonal decomposition is implemented using filter banks.

The implementations of the orthogonal decomposition using linear and quadratic piecewise polynomial scaling and wavelet bases showed that the main important classical characteristics of the traditional multiresolution approach (i.e. regular spaced samples) are still maintained. Among these characteristics, the provided simulations illustrated that (i) the smallest variance of the residual signal is obtained when there is a decrease in the resolution and (ii) increasing the spline function degree results in a decrease of the variance of the residual signal. Once this theory has been explained, one can now use the corresponding framework for traditional applications such as denoising, compression.

Among the proposed wavelet functions, the particular one with regularity $C^{d-1}$ and equal values at the endpoints of the interval presents many interesting and desirable properties in the specific context of uniformly spaced data. Indeed this wavelet is compactly supported, symmetrical and its regularity can be controlled almost everywhere when acting on the spline function degree. It would be quite interesting to study the features of the corresponding filter banks in future investigations.

\section{References}

[1] S. Mallat, A Wavelet Tour of Signal Processing, Academic Press, San Diego, Calif, USA, 1999.

[2] M. Vetterli and J. Kovacevic, Wavelets and Subband Coding, Prentice Hall, Upper Saddle River, NJ, USA, 1995.

[3] C. K. Chui, An Introduction to Wavelets, vol. 1 of Wavelet Analysis and Its Applications, chapter 6, Academic Press, New York, NY, USA, 1992.

[4] O. Rioul and P. Duhamel, "Fast algorithms for wavelet transform computation," in Time-Frequency and Wavelets in Biomedical Engineering, M. Akay, Ed., chapter 8, pp. 211-242, IEEE Press, New York, NY, USA, 1997.

[5] G. Farin, Curves and Surfaces for CAGD: A Practical Guide, Morgan-Kaufmann, San Francisco, Calif, USA, 5th edition, 2002.

[6] M. Unser, "Splines: a perfect fit for signal and image processing," IEEE Signal Processing Magazine, vol. 16, no. 6, pp. 22-38, 1999.

[7] M. Unser, A. Aldroubi, and M. Eden, "B-spline signal processing-part I: theory," IEEE Transactions on Signal Processing, vol. 41, no. 2, pp. 821-833, 1993.

[8] M. Unser, A. Aldroubi, and M. Eden, "B-spline signal processing-part II: efficient design and applications," IEEE Transactions on Signal Processing, vol. 41, no. 2, pp. 834-848, 1993.

[9] P. Thévenaz, T. Blu, and M. Unser, "Interpolation revisited," IEEE Transactions on Medical Imaging, vol. 19, no. 7, pp. 739$758,2000$.

[10] M. Unser, “Ten good reasons for using spline wavelets," in Proceedings of the 4th Conference on Mathematical Imaging: Wavelet Applications in Signal and Image Processing, vol. 3169, pp. 422-431, San Diego, Calif, USA, July 1997.

[11] I. Daubechies, I. Guskov, P. Schröder, and W. Sweldens, "Wavelets on irregular point sets," Philosophical Transactions of the Royal Society of London A, vol. 357, no. 1760, pp. 23972413, 1999.

[12] I. Daubechies, I. Guskov, and W. Sweldens, "Regularity of irregular subdivision," Constructive Approximation, vol. 15, no. 3, pp. 381-426, 1999.

[13] I. Daubechies, I. Guskov, and W. Sweldens, "Commutation for irregular subdivision," Constructive Approximation, vol. 17, no. 4, pp. 479-514, 2001.

[14] T. Lyche, K. Mørken, and E. Quak, "Theory and algorithms for non-uniform spline wavelets," in Multivariate Approximation and Applications, N. Dyn, D. Leviatan, D. Levin, and A. Pinkus, Eds., pp. 152-187, Cambridge University Press, Cambridge, UK, 2001.

[15] M. D. Buhmann and C. A. Micchelli, "Spline prewavelets for non uniform knots," Numerical Mathematics, vol. 61, pp. 455474, 1992.

[16] M. Bertram, "Single-knot wavelets for non-uniform Bsplines," Computer Aided Geometric Design, vol. 22, no. 9, pp. 849-864, 2005.

[17] J. Wang, "Interpolating cubic spline wavelet packet on arbitrary partitions," Journal of Computational Analysis and Applications, vol. 5, pp. 179-193, 2003.

[18] N. Chihab, A. Zergaïnoh, P. Duhamel, and J.-P. Astruc, "The influence of the non-uniform spline basis on the approximation signal," in Proceedings of the 12th European Signal Processing Conference, Vienna, Austria, September 2004.

[19] M. Lounsbery, T. DeRose, and J. Warren, "Multiresolution analysis for surfaces of arbitrary topological type," ACM Transactions on Graphics, vol. 16, no. 1, pp. 34-73, 1997. 
[20] A. Zergainoh, N. Chihab, and J.-P. Astruc, "Construction of orthonormal piecewise polynomial scaling and wavelet bases on non-equally spaced knots," EURASIP Journal on Advances in Signal Processing, vol. 2007, Article ID 27427, 13 pages, 2007.

[21] C. De Boor, A Practical Guide to Splines, Springer, Berlin, Germany, 2001.

[22] H. B. Curry and I. J. Schoenberg, "On spline distributions and their limits: The polya distribution functions," Bull. Amer. Math, vol. 53, no. 1114, 87 pages, 1947. 